\title{
MULTIVARIATE MODELING AND ANALYSIS OF REGIONAL OCEAN FREIGHT RATES
}

\begin{abstract}
In this paper, we propose a new multivariate model for the dynamics of regional ocean freight rates. We show that a cointegrated system of regional spot freight rates can be decomposed into a common non-stationary market factor and stationary regional deviations. The resulting integrated CAR process is new to the literature. By interpreting the common market factor as the global arithmetic average of the regional rates, both the market factor and the regional deviations are observable which simplifies the calibration of the model. Moreover, forward contracts on the market factor can be traded in the Forward Freight Agreement (FFA) market. We calibrate the model to historical spot rate processes and illustrate the term structures of volatility and correlation between the regional prices and the market factor. Our model is an important contribution towards improved modelling and hedging of regional price risk when derivative market liquidity is concentrated in a single global benchmark.
\end{abstract}

\section{INTRODUCTION}

The degree to which regional markets for a homogeneous good are spatially integrated across the globe will depend on the physical characteristics of international trade. The greater the trade barriers in terms of time, transport costs, and tariffs, the less integrated are regional market prices. Ocean transportation is an integral part of this picture, particularly in the global commodity markets, as the vast majority of trade is seaborne. The fact that ships and their cargoes move slowly around the world compared to other transportation modes (Hummels and Schaur [27]) implies that regional imbalances in the physical supply and demand of a commodity cannot be immediately resolved by international trade. Yet, whenever two regional prices deviate by more than the transaction costs, commodity traders will take advantage of the spatial price arbitrage by shipping cargoes from the cheaper region to the expensive region such that prices are realigned (Pirrong [39]). Large spatial differences in regional freight rates will have a similar effect on the physical movement of ships, as profit-maximizing shipowners will reallocate their fleet to higher-paying areas, thereby realigning regional freight rates (Adland et al. [1]). The topic of spatial market integration has been investigated empirically across many global commodity markets, notably coal (Wårell [48]; Papiez and Smiech [38]), natural gas (Siliverstovs et al. [44]), crude oil (Bachmeier and Griffin [4]), petroleum products (Lanza et al [34]), and regional ocean freight rates (Berg-Andreassen [16, 17]; Glen and Rogers [26]; Veenstra and Franses [47]). The general finding is that regional prices are nonstationary and co-integrated. Following the definition of Engle and Granger [25]), this means the time series are integrated, or I(1), while some linear combination between them - the cointegrating vector - is stationary, or $\mathrm{I}(0)$. This is consistent with the idea that regional prices must revert towards some common global stochastic trend because of the ability of cargoes and ships to continuously move from regions of oversupply towards regions of undersupply.

Having established that regional prices or freight rates are co-integrated, usually based on the test methodology of Johansen [28], most of the above studies proceed to model them jointly in the Vector Error Correction (VEC) model framework (see, for instance, Veenstra and Franses [47]; Lanza et al. [34]). An alternative approach is the state space representation, where the common stochastic process is unobservable and extracted empirically using, for instance, the Kalman filter approach (Chang et al. [19]). Ko [31, 32] uses the latter methodology to derive a common stochastic trend from average freight rate indices for different vessel sizes in the drybulk freight market and assess the idiosyncratic dynamics of size effects.

For the purposes of risk management, there are numerous regional spot freight rates for drybulk carriers, but liquidity in the derivative market is concentrated in Forward Freight Agreements (FFAs) written on the

Date: Monday $18^{\text {th }}$ September, 2017.

Key words and phrases. Freight rates, autoregressive models in continuous time, cointegration, Forward freight agreements.

We are grateful to Clarkson Research for provding us with data. The authors acknowledge financial support from the research project "Green shipping under uncertainty" partly financed by the Research Council of Norway under grant number 233985. We thank three referees for their valuable and constructive criticism on an earlier version of the paper. 
weighted global average of such indices. A similar situation exists in the crude oil and fuel oil market, with a large number of regional prices available around the globe but derivative liquidity focused on one or two global benchmarks (e.g. Brent crude or Rotterdam HFO). In the context of cross hedging, the implementation and interpretation of a model becomes considerably easier if the common stochastic factor explicitly represents an observable market price on which tradable derivatives exist. In this paper we propose, for the first time, to decompose regional prices into such a common observable stochastic factor and mean-reverting, potentially correlated, regional factors. This factorization can be viewed as an extension of the famous model by Schwartz and Smith [43], which is the price dynamics empirically argued for by Prokopczuk [40] in his seminal work on freight futures pricing and hedging.

The contribution of our paper is threefold. Firstly, we develop a new continuous-time stochastic model for the joint dynamics of regional prices, the Integrated Continuous Autoregressive, or ICAR, process. Our proposed framework enables the decomposition of observed regional spot price dynamics into a nonstationary observable market factor, for which tradable contracts exist, and observable stationary regional factors (deviations from the market average). We focus on Gaussian models, but provide also some theoretical discussion and empirical evidence for leptokurtic dynamic models. Secondly, we show the link between the continuous ARMA process and the ARMA time series model, generalizing results for the specific autoregressive case in Benth and Šaltyte Benth [12]. Thirdly, we calibrate the model empirically against regional spot freight processes in the Supramax market in both discrete and continuous time, and illustrate the resulting term structures of correlations and volatility versus contract maturity.

Our findings are important for shipping industry players and maritime economic researchers alike. Notably, we bring new insight into how to model and estimate the joint dynamics of regional spot rates in both a discrete and continuous-time setting. The ease of estimation and simulation makes the model particularly interesting for stochastic scenario generation, which would form an important input in optimization models for fleet allocation (i.e. how to optimally sequence tripcharters in the bulk shipping spot market through space and time). In the context of risk management, our extension to the freight derivatives market directly addresses a practical question that all shipowners, operators and charterers trading drybulk freight derivatives must deal with: how to hedge physical regional exposure when only global averages are tradable in practice. Here, our model also brings the literature on freight derivatives forward by illustrating the implication of our model for the pricing of regional forward curves and the term structure of volatility.

The remainder of our paper is structured as follows: Section 2 derives the continuous-time cointegrated model of the spot freight rate dynamics, Section 3 presents our data and time series analysis, Section 4 derives the theoretical FFA price dynamics within our framework, Section 5 shows an application to the hedging of regional risk and Section 6 gives an outlook to non-Gaussian dynamical models. Finally, Section 7 concludes.

\section{A CONTINUOUS-TIME COINTEGRATED MODEL FOR THE FREIGHT RATE SPOT DYNAMICS}

In this Section we develop a continuous-time stochastic model for the dynamics of freight rates. To distinguish continuous-time stochastic processes from time series, we will use the notation $Y(t)$ for a continuous time process, while we apply the notation $y_{t}$ for a time series model (with time $t$ in that case being discrete). In this section and in the remainder of the paper, we let $(\Omega, \mathcal{F}, P)$ define a complete probability space, equipped with a filtration $\left\{\mathcal{F}_{t}\right\}_{t \geq 0}$.

First, recall from Brockwell [18] that a real-valued stochastic process $Y$ is called a $\operatorname{CAR}(p)$-process for $p \in \mathbb{N}$, if $Y(t)=\mathbf{e}_{1}^{\top} \mathbf{Z}(t)$ where $\mathbf{Z} \in \mathbb{R}^{p}$ is the vector-valued Ornstein-Uhlenbeck process given by

$$
d \mathbf{Z}(t)=A \mathbf{Z}(t) d t+\sigma \mathbf{e}_{p} d B(t), \quad \mathbf{Z}(0)=\mathbf{Z}^{0} \in \mathbb{R}^{p},
$$

for a Brownian motion $B$. Here, $\left\{\mathbf{e}_{k}\right\}_{k=1}^{p} \subset \mathbb{R}^{p}$ is the canonical basis of $\mathbb{R}^{p}$ and $\sigma>0$ is a constant. The matrix $A \in \mathbb{R}^{p \times p}$ is defined as

$$
A=\left[\begin{array}{cccccc}
0 & 1 & 0 & 0 & \ldots & 0 \\
0 & 0 & 1 & 0 & \ldots & 0 \\
. & . & . & . & \ldots & . \\
. & . & . & . & \ldots & . \\
0 & 0 & 0 & 0 & \ldots & 1 \\
-\alpha_{p} & -\alpha_{p-1} & -\alpha_{p-2} & -\alpha_{p-3} & \ldots & -\alpha_{1}
\end{array}\right]
$$


for positive constants $\alpha_{k}, k=1, \ldots, p$. Hence,

$$
Y(t)=\mathbf{e}_{1}^{\top} e^{A t} \mathbf{Z}^{0}+\sigma \int_{0}^{t} \mathbf{e}_{1}^{\top} e^{A(t-s)} \mathbf{e}_{p} d B(s) .
$$

It is known that the distribution of $Y(t)$ converges when $t \rightarrow \infty$ to a normal distribution with mean zero and variance

$$
\operatorname{Var}[Y(t)]=\mathbf{e}_{p}^{\top} \int_{0}^{\infty} e^{A^{\top} s} \mathbf{e}_{1} \mathbf{e}_{1}^{\top} e^{A s} d s \mathbf{e}_{p},
$$

if and only if the eigenvalues of $A$ have strictly negative real part. The (strictly) stationary representation of $Y$ is given as

$$
Y(t)=\int_{-\infty}^{t} \mathbf{e}_{1}^{\top} e^{A(t-s)} \mathbf{e}_{p} d B(s) .
$$

We will frequently refer to $A$ as the $C A R$-matrix. If the eigenvalues of $Y$ all have strictly negative real part, we say that $Y$ is stationary. The process $Y$ is a continuous-time version of a autoregressive process of order $p$ (see Brockwell [18]).

One can easily extend continuous-time autoregressive processes to include a moving average feature as well. With $q \in \mathbb{N}, q<p$, a $\operatorname{CARMA}(p, q)$ process is defined, following Brockwell [18], by

$$
Y(t)=\mathbf{b}^{\top} \mathbf{Z}(t)
$$

for $\mathbf{b} \in \mathbb{R}^{p}$, where $\mathbf{b}=\left(b_{0}, b_{1}, \ldots, b_{q}, 0, \ldots, 0\right)^{\top}$ and $b_{q}=1$. We observe that for $q=0$ we recover the definition of a $\operatorname{CAR}(p)$ process. It is simple to see that the characteristic polynomial of the CAR-matrix $A$ in (2), denoted $P(\lambda)$, is

$$
P(\lambda)=\lambda^{p}+\alpha_{1} \lambda^{p-1}+\cdots+\alpha_{p-1} \lambda+\alpha_{p},
$$

for $\lambda \in \mathbb{C}$. We also introduce the $q$ th-order polynomial $Q(\lambda)$,

$$
Q(\lambda)=b_{0}+b_{1} \lambda+b_{2} \lambda^{2}+\cdots+b_{q} \lambda^{q},
$$

for $\lambda \in \mathbb{C}$. Indeed, we have the representation (see Brockwell [18])

$$
P(D) Y(t)=Q(D) D B(t)
$$

for a $\operatorname{CARMA}(p, q)$-process, where $D=d / d t$ is the derivative operator. Of course, this is a formal representation, as the time derivative of Brownian motion is not well-defined. However, from (8) we can show a link between $\operatorname{CARMA}(p, q)$ processes and time series of $\operatorname{ARMA}(p, q)$ type. This link is established using a so-called Euler approximation of the dynamics of $Y$ based on the representation in (8), with $D$ being approximated by forward differences. Recall the $n$th order forward differencing operator for a time step $\delta>0$

$$
\Delta_{\delta}^{n} f(t)=\sum_{k=0}^{n}\left(\begin{array}{l}
n \\
k
\end{array}\right)(-1)^{k} f(t+(n-k) \delta)
$$

acting on a function $f: \mathbb{R} \rightarrow \mathbb{R}$. Then $\delta^{-n} \Delta_{\delta}^{n}$ is a numerical approximation of the $n$th time derivative. Let us now introduce the time series $\left\{y_{i}\right\}_{i=0}^{\infty}$, and consider

$$
P\left(\delta^{-1} \Delta_{\delta}\right) y_{i}=Q\left(\delta^{-1} \Delta_{\delta}\right) \delta^{-1} \Delta_{\delta} B\left(t_{i}\right)
$$

where $t_{i}:=i \delta, i=0, \ldots$ and use the convention that $y_{i}=y\left(t_{i}\right)$ in the forward differencing. We have the following proposition (with the proof relegated to the Appendix):

Proposition 1. The time series $\left\{y_{i}\right\}_{i=0}^{\infty}$ is $\operatorname{ARMA}(p, q)$,

$$
y_{i+p}=\sum_{k=1}^{p} \beta_{k} y_{i+p-k}+\sum_{n=0}^{q} \eta_{n} \tilde{\epsilon}_{i+n}
$$

with

$$
\beta_{k}=(-1)^{k+1}\left(\begin{array}{l}
p \\
k
\end{array}\right)-\sum_{j=1}^{k} \alpha_{j} \delta^{j}(-1)^{k-j}\left(\begin{array}{l}
p-j \\
k-j
\end{array}\right), k=1, \ldots, p
$$




$$
\eta_{n}=\sum_{j=n}^{q} b_{j} \delta^{p-j}(-1)^{j-n}\left(\begin{array}{c}
j \\
j-n
\end{array}\right), n=0, \ldots, q,
$$

and $\left\{\widetilde{\epsilon}_{i}\right\}_{i=0}^{\infty}$ is a series of IID mean-zero normally distributed random variables with variance $\sigma^{2} / \delta$.

This result is a generalization of the analysis in Benth and Šaltytė Benth [12, Sect. 4.3], where the particular relationship between a $\operatorname{CAR}(p)$-dynamics and an $\operatorname{AR}(p)$ time series were established. Obviously, to let the recursion in Proposition 1 run, we must assume that $y_{0}, \ldots, y_{p-1}$ are given.

In the following, we will exclusively restrict our attention to $\operatorname{CAR}(p)$ processes since these are the relevant ones from the empirical analysis, as we will see in the next section.

For $n \in \mathbb{N}$, let $\mathbf{Y}=\left(Y_{0}, Y_{1}, \ldots, Y_{n}\right)^{\top} \in \mathbb{R}^{n+1}$ be a vector valued CAR-process defined as follows: for $\mathbf{p}=\left(p_{0}, p_{1}, \ldots, p_{n}\right)^{\top}$, let $Y_{i}$ be CAR $\left(p_{i}\right)$-process for $i=0,1, \ldots, n$ with CAR-matrix $A_{i}$, volatility $\sigma_{i} \in \mathbb{R}_{+}$and finally Brownian motion $B_{i}$. We suppose that $\mathbf{B}=\left(B_{0}, B_{1}, \ldots, B_{n}\right)^{\top}$ is a $n+1$-dimensional Brownian motion, with possibly correlated coordinate processes, and refer to $\mathbf{Y}$ as a CAR(p)-process.

We model a market of $n$ routes by the following continuous-time cointegrated dynamics: The freight rate dynamics of route $i, i=1, \ldots, n$, is denoted by $S_{i}$ and defined by

$$
\ln S_{i}(t)=X(t)+Y_{i}(t)
$$

with

$$
X(t)=\int_{0}^{t} Y_{0}(s) d s .
$$

The common factor $X$ will become, as we shall see, a non-stationary stochastic process. Indeed, we show below that it is an integrated autoregressive process. The second factor in the log-price of rute $i, Y_{i}$, is a stationary autoregressive moving average process, and one can therefore view the dynamics in (10) as a generalization of the two-factor model of Schwartz and Smith [43]. The generalization goes in two directions, one being an extension of the dynamics of the two factors and the other to define the model in a cointegration context. The dynamics of all the regional routes can be expressed by the vector-valued process $\mathbf{S}=\left(S_{1}, \ldots, S_{n}\right)^{\top} \in \mathbb{R}^{n}$.

Let us study the process $X$ in (11).

Proposition 2. The process $X$ defined in (11) is Gaussian with mean

$$
\mathbb{E}[X(t)]=\mathbf{e}_{1}^{\top} A_{0}^{-1}\left(e^{A_{0} t}-I\right) \mathbf{Z}_{0}^{0},
$$

for $\mathbf{Z}_{0}^{0}$ being the initial state of $\mathbf{Z}_{0}(t)$, and variance

$$
\operatorname{Var}[X(t)]=2 \sigma_{0}^{2} \mathbf{e}_{p_{0}}^{\top} \int_{0}^{t} \int_{0}^{s} e^{A_{0}^{\top}(s-u)}\left(\int_{0}^{u} e^{A_{0}^{\top} v} \mathbf{e}_{1} \mathbf{e}_{1}^{\top} e^{A_{0} v} d v\right) d u d s \mathbf{e}_{p_{0}} .
$$

From Lemma 4.2 in Benth and Šaltytė Benth [12], the inverse of a general CAR-matrix $A$ defined in (2) is explicitly available. Therefore, we know $A_{0}^{-1}$ in the expression for the mean of $X$ in Proposition 2.

In the next Proposition we show that $X$ in (11) is a non-stationary $\operatorname{CAR}\left(p_{0}+1\right)$-process.

Proposition 3. The process $X$ in $(11)$ is a $C A R\left(p_{0}+1\right)$-process with a CAR-matrix $\bar{A}_{0}$ given by

$$
\bar{A}_{0}=\left[\begin{array}{cc}
0 & \mathbf{e}_{1}^{\top} \\
\mathbf{0} & A_{0}
\end{array}\right]
$$

where $\mathbf{0} \in \mathbb{R}^{p_{0}}$ is the zero vector. The matrix $\bar{A}_{0}$ has zero as an eigenvalue.

Remark in passing that we have, strictly speaking, only defined a CAR-matrix in the case when the elements in the last row are all negative. In the above representation, we dispense with this and include also the case of zero values, as turns out to be appropriate to facilitate the representation of $X$. Since zero is an eigenvalue of $\bar{A}_{0}$, at least one eigenvalue of the CAR $\left(p_{0}+1\right)$-process $X$ does not have a strictly negative real part, and we conclude that its distribution cannot have a limit as $t \rightarrow \infty$. Hence, it is non-stationary. Notice from Proposition 2 that if $Y_{0}$ is a stationary $\operatorname{CAR}\left(p_{0}\right)$-process, then the mean of $X(t)$ converges to $-\mathbf{e}_{1} A_{0}^{-1} \mathbf{Z}_{0}^{0}$. However, the variance will diverge. For example, consider $p_{0}=1$ when $Y_{0}$ becomes an 
Ornstein-Uhlenbeck process with speed of mean reversion $\alpha_{0}$. I.e., $A_{0}=-\alpha_{0}$ and from Proposition 2 we find

$$
\operatorname{Var}[X(t)]=\frac{\sigma_{0}^{2}}{2 \alpha_{0}}\left(t-\frac{2}{\alpha_{0}}\left(1-e^{-\alpha_{0} t}\right)+\frac{1}{2 \alpha_{0}}\left(1-e^{-2 \alpha_{0} t}\right)\right) .
$$

Therefore, $\operatorname{Var}(X(t)) \sim k t$ when $t \rightarrow \infty$ for $k=\sigma_{0}^{2} / 2 \alpha_{0}$, and we conclude that the variance diverges and $X$ is non-stationary.

Recall our freight rate dynamics, and observe that

$$
\mathbf{c}^{\top} \log \mathbf{S}=\sum_{i=1}^{n} c_{i} Y_{i}
$$

for any $\mathbf{c} \in \mathbb{R}^{n}$ such that $\sum_{i=1}^{n} c_{i}=0$. For example, if $n$ is even, we can choose $\mathbf{c}=(1,-1,1,-1, \ldots .1,-1)^{\top}$. If $Y_{i}, i=1, \ldots, n$ are stationary CAR-processes, we conclude that $\mathbf{S}$ is a cointegrated model. Straightforwardly we see that for any $i, j \in\{1,2, \ldots, n\}$,

$$
\ln S_{i}(t)-\ln S_{j}(t)=Y_{i}(t)-Y_{j}(t),
$$

and thus any pair of routes are cointegrated.

We next show that the process $X$ can be viewed as an integrated $\operatorname{AR}\left(p_{0}\right)$ process in discrete time. To this end, let $q=0$ to yield the polynomial $Q(\lambda)=1$ in (7), and define the time series $\left\{x_{i}\right\}_{i=0}^{\infty}$ given by (9) for the polynomial $P(\lambda)$ associated with the CAR-matrix $\bar{A}_{0}$ defined in Proposition 3 . Finally, define $\Delta x_{i}:=x_{i+1}-x_{i}$, to find,

Proposition 4. It holds that $\left\{x_{i}\right\}_{i=0}^{\infty}$ is an integrated $A R\left(p_{0}\right)$ time series with

$$
\Delta x_{i+p_{0}}=\sum_{k=1}^{p_{0}} \xi_{k} \Delta x_{i+p_{0}-k}+\sigma \delta^{p_{0}+1 / 2} \epsilon_{i} .
$$

Here, $\left\{\epsilon_{i}\right\}_{i=0}^{\infty}$ are IID standard normal random variables and $\xi_{k}, k=1, \ldots, p_{0}$ are defined recursively by $\xi_{1}=\beta_{1}-1$ and $\xi_{k}=\xi_{k-1}+\beta_{k}$ for $k=2, \ldots, p_{0}$ for

$$
\beta_{k}=(-1)^{k+1}\left(\begin{array}{c}
p_{0}+1 \\
k
\end{array}\right)-\sum_{j=1}^{j} \alpha_{j} \delta^{j}(-1)^{k-j}\left(\begin{array}{c}
p_{0}+1-j \\
k-j
\end{array}\right), k=1, \ldots, p_{0},
$$

and $\alpha_{1}, \ldots \alpha_{p_{0}}$ comes from the CAR-matrix $A_{0}$.

This result motivates the name integrated $\operatorname{CAR}\left(p_{0}\right)$, or $\operatorname{ICAR}\left(p_{0}\right)$ for short, associated with the process $X$.

Kavussanos and Alizadeh [30] investigate empirically the nature of seasonality in spot and TC rates in the drybulk market across different sizes. They find significant deterministic seasonality at the monthly level, with asymmetric and market-dependent seasonal fluctuations. We do not explicitly consider seasonality in our model and analysis to maintain a parsimonious model and to avoid the possible biases introduced by de-seasonalizing spot freight rates using deterministic monthly factors when these are in fact time-varying and market dependent. This is particularly critical as we consider regional rates in our analysis, which may be more sensitive to such adjustments than the global average spot rates considered in the literature.

\section{DATA DESCRIPTION AND TIME SERIES ANALYSIS}

As the empirical case for our regional freight rate model we pick the market for Supramax bulk carriers. Supramax vessels are mid-size drybulk vessels of about 52,000 DWT capacity that carry a wide range of drybulk commodities around the world, principally coal, iron ore, grains, soybeans and steel products. The physical dimensions of the vessels are such that they can be accepted by most ports, leading to a diverse trading pattern. However, based on the intercontinental trade of drybulk commodities within and between the two main ocean basins (Atlantic and Pacific) we can broadly group the trade flows into four main region-to-region routes: i) Atlantic to Pacific, ii) trans-Pacific, iii) Pacific to Atlantic, and iv) trans-Atlantic. Following the standard terminology in the industry, the Atlantic-to-Pacific trade is termed Fronthaul and the reverse trade Backhaul (see Alizadeh and Nomikos [2]), where Fronthaul refers to higher cargo volumes 


\begin{tabular}{cl} 
Route & Route description \\
\hline 1 & BSI Route S1: Fronthaul \\
2 & BSI Route S2: Japan-South Korea/NOPAC or Australia RV \$/Day \\
3 & BSI Route S3: Japan-SK Trip Gib-Skaw range \$/Day \\
4 & BSI Route S4: Transatlantic RV \\
\hline
\end{tabular}

TABLE 1. Supramax routes (RV stands for Round Voyage)

and generally higher freight rates. Using weekly Baltic spot freight rate data (\$/day vessel hire) obtained from Clarkson Research [20] results in $N=540$ observations for the period 2007-2015 for each route.

Formally, with reference to the standard route definitions in Baltic Exchange [6], we focus on regional freight rates for the four routes detailed in Table $1 .{ }^{1}$ Additionally, we refer to the Supramax timecharter average as the equal-weighted (25\% each) arithmetic average of the freight rates for the individual routes. The Supramax average is the spot price index against which Forward Freight Agreements (FFAs) for this vessel size are settled (see Alizadeh and Nomikos [2]).

Let $S_{i, t}$ represent the freight rate at time $t$ for route $i=1,2,3,4$, and let $s_{i, t}$ represent the logarithm of the freight rate $S_{i, t}$, that is, we assume the relationship

$$
S_{i, t}=\exp \left(s_{i, t}\right) .
$$

The respective time series are plotted in Figure 1. Route 1 and 4 have higher maximum values compared to routes 2 and 3 (indeed, also higher mean as seen from the descriptive statistics in Table 2). Neither route deviates too far from the others as time evolves, even in periods with very high volatility. From Figure 1 there seems to be two distinct periods: Increasing freight rates in 2005-2007 and low freight rates in 20092015. Inbetween these two periods, we observe that 2008 was a "rollercoaster year": First a decline, then an increase where $S_{4}$ sets a maximum spot of 94,718 \$/day, and finally a steep decline in the second part of the year along with the onset of the global financial crisis. We also note that the individual routes stay closer together in the first period, but somewhat less so in the second period, see especially the lower panel with logarithmic rates.
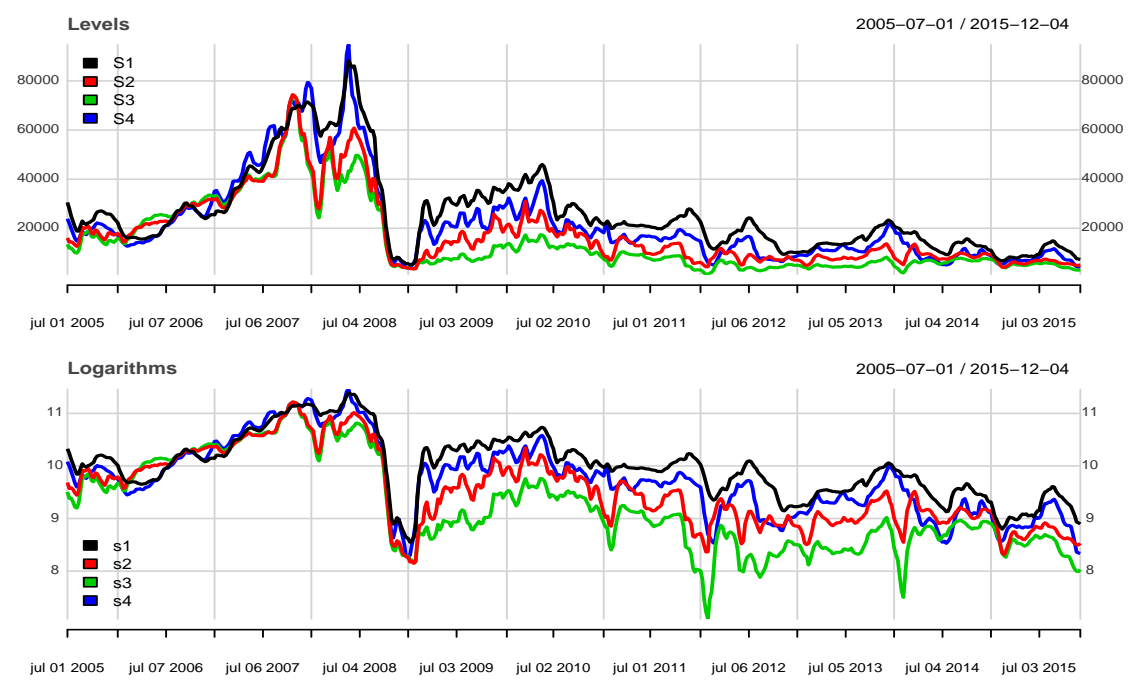

FIGURE 1. Supramax spot freight rates for four market segments. Upper panel: Data in levels. Lower panel: Data in logarithms

Our ultimate goal is to propose a model for the joint freight rate dynamics, both in discrete and continuous time, but we start out by looking at the properties of individual time series. In the following we will

\footnotetext{
${ }^{1}$ Note that in this table S1 represents the arithmetic average of two Fronthaul trips (S1A and S1B), while S4 the arithmetic average of the Atlantic westbound and eastbound trips (S4A and S4B)
} 
focus on the logarithmic spot rates. Just by looking at Figure 1 we note the strong persistence in the data, with the mean of the series changing over time. This is confirmed by the autocorrelation plots in Figure 2 . The shaded area in the graphs is the $95 \%$ level of significance. The estimated autocorrelation functions for the logarithmic spot rates all show very slow decay, indicating that the data is non-stationary. Since we suspect non-stationary time series, we next proceed with unit root tests.
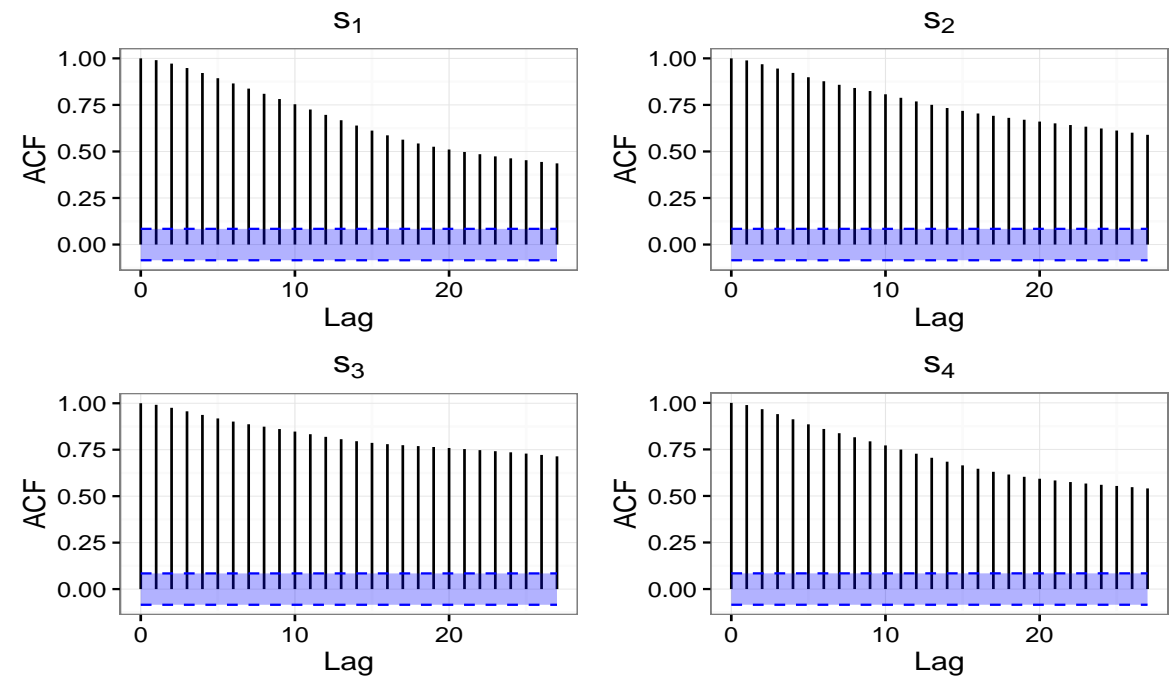

FIGURE 2. Autocorrelation plots for logarithmic freight rate data for each route.

Consider the following version of the Augmented Dickey-Fuller test (see Dickey and Fuller [21] and Said and Dickey [42]): introduce a time series $\left\{u_{t}\right\}_{t=1, \ldots}$ given by

$$
\Delta u_{t}=d_{0}+\beta_{0} u_{t-1}+\sum_{j=1}^{k} \beta_{j} \Delta u_{t-j}+\epsilon_{t},
$$

where $d_{0}$ is a constant term, $\beta_{0}, \beta_{1}, \ldots, \beta_{k}$ are parameters, $\Delta$ is the lag operator, that is, $\Delta u_{t}=u_{t}-u_{t-1}$ and finally $\left\{\epsilon_{t}\right\}_{t \in \mathbb{N}}$ is IID noise. The unit root test is a one-tailed $t$-test on the parameter $\beta_{0}=0$ against the stationary alternative $\beta_{0}<0$. Standard unit root tests of Dickey and Fuller [21] have very low power against stationary near unit-root alternatives. For maximum power against very persistent alternatives the so-called efficient unit root tests proposed by Elliot et al. [24] could be used. Efficient unit root tests follow a two-step procedure; first de-trending the data using generalized least squares (GLS) and then performing a so called point-optimal test on the de-trended data. They also proposed a modified ADF-test based on GLS de-trending. We conduct both the feasible point optimal test (PT) and the modified ADF-test (ADFGLS). We use the method of $\mathrm{Ng}$ and Perron [35] and the BIC criteria for lag length selection in the GLS de-trending step. Test statistics and lag selection for the different routes are presented in Table 2 below. From the left panel, we see that the null hypothesis of non-stationarity cannot be rejected for the logarithm of freight rates for routes 1,3 and 4 . Route 2 is marginally significant on the $5 \%$ level of significance, indicating a rejection of the null hypothesis of non-stationarity. In the right panel of Table 2 we report test statistics for the same tests, but now on the data in first differences. The ADF-GLS test rejects the null hypothesis of non-stationarity on $1 \%$ level of significance, and accepts the stationary alternative. The same conclusion can be drawn from the PT test, but on 5\% level of significance for routes 2 and 3. Overall, we conclude that the logarithm of Supramax spot rates are non-stationary, and that the time series must be differenced once to achieve stationarity. This is consistent with findings in the literature, where spot rates are usually found to be a non-stationary process (see, for instance, Berg-Andreassen [16], Glen and Rogers [26] and Kavussanos and Alizadeh [30]).

Next we investigate the empirical properties of the logarithm of the spot rates in first differences. In Figure 3 and 4 we present the empirical autocorrelation plots and partial autocorrelation plots, respectively. 
MULTIVARIATE MODELING AND ANALYSIS OF REGIONAL OCEAN FREIGHT RATES

\begin{tabular}{lcccccccc} 
& Data in logarithms & \multicolumn{5}{c}{ Data in logarithmic differences } \\
\hline & $s_{1}$ & $s_{2}$ & $s_{3}$ & $s_{4}$ & $\Delta s_{1}$ & $\Delta s_{2}$ & $\Delta s_{3}$ & $\Delta s_{4}$ \\
\hline Mean & 10.0 & 9.5 & 9.2 & 9.7 & -0.003 & -0.002 & -0.003 & -0.003 \\
Median & 10.0 & 9.5 & 9.0 & 9.7 & -0.001 & -0.001 & -0.003 & -0.001 \\
Min & 8.6 & 8.1 & 7.1 & 8.2 & -0.353 & -0.392 & -0.399 & -0.469 \\
Max & 11.4 & 11.2 & 11.2 & 11.5 & 0.587 & 0.486 & 0.473 & 0.538 \\
\hline ADF-GLS & -1.96 & $-2.01^{*}$ & -1.81 & -1.87 & $-3.33^{* *}$ & $-2.76^{* *}$ & $-2.75^{* *}$ & $-6.65^{* *}$ \\
PT & 3.38 & $3.17^{*}$ & 3.76 & 3.67 & $1.3^{* *}$ & $2.46^{*}$ & $2.47^{*}$ & $0.31^{* *}$ \\
Lags & 4 & 2 & 2 & 2 & 6 & 8 & 8 & 2 \\
\hline
\end{tabular}

TABLE 2. Descriptive statistics and unit root tests for Supramax rates in logarithms and logarithmic first differences. Critical levels for the ADF-GLS test on $1 \%$ and $5 \%$ level of significance is -2.58 and -1.98 , respectively. Critical levels for PT test on $1 \%$ and $5 \%$ level of significance is 1.78 and 3.17 , respectively. The BIC criterion is used for lag length selection.
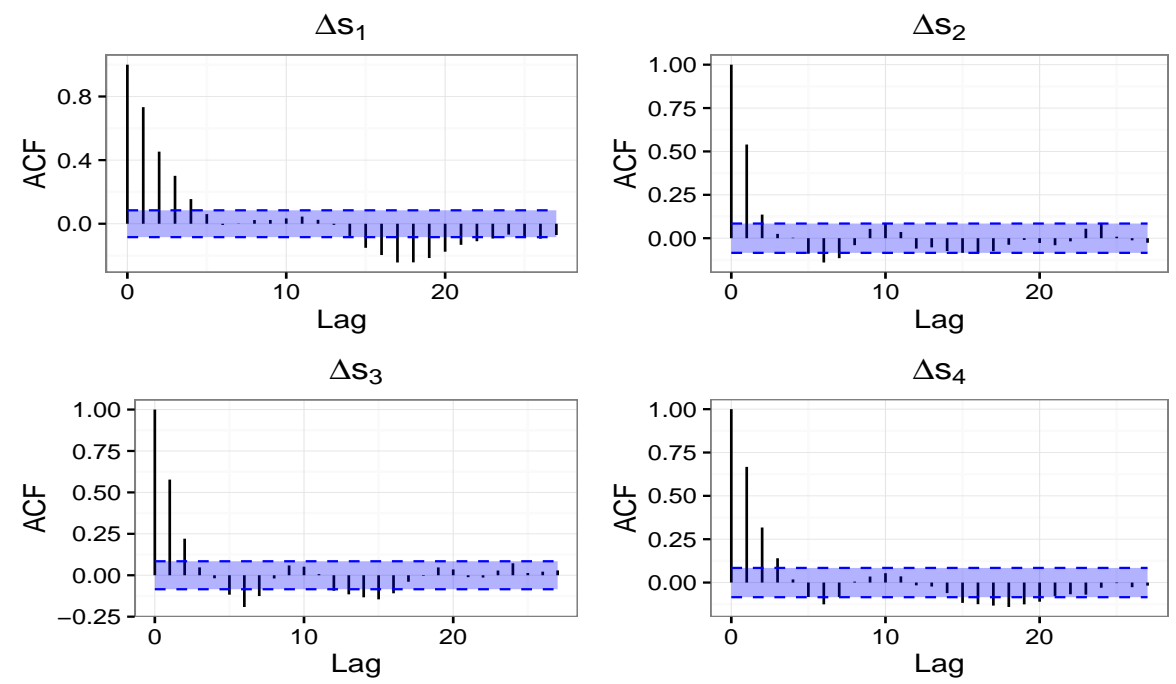

FIGURE 3. Autocorrelation plots for logarithmic freight rate data in first differences.

Unsurprisingly, the patterns for the (partial) autocorrelations are similar across routes. The decay in the autocorrelations in Figure 3 is much more rapid compared to the decay in autocorrelations in Figure 2. This is of course consistent with the unit root test results above. The partial autocorrelations in Figure 4 show that the first two lags are significant for all four routes; the first lag being positive, and second negative. As an initial modelling effort we fit a higher order autoregressive model (AR $(p)$-model) for each route. We apply the BIC criterion for selecting the appropriate number of lags. This resulted in an AR(2) model for each series,

$$
\Delta s_{i, t}=\beta_{1, i} \Delta s_{i, t-1}+\beta_{2, i} \Delta s_{i, t-2}+\epsilon_{i, t},
$$

with estimated parameters given in Table 3. From Figure 1 we know that the freight rates stay close together in the sample period, an indication that the rates are strongly correlated. Indeed, Table 4 presents estimated correlations of the residuals from the fitted $\mathrm{AR}(2)$ models. We note that route 2 and 3 show the strongest degree of correlation of 0.83 , while route 1 and 3 show the lowest degree of correlation of 0.43 .

At this stage, one might be tempted to model the joint freight dynamics as a multivariate AR(2)-model for logarithmic differences with correlated residuals. But this is unfortunately not very fruitful. Correlated nonstationary processes will tend to move away from each other over time. When two series have drifted 

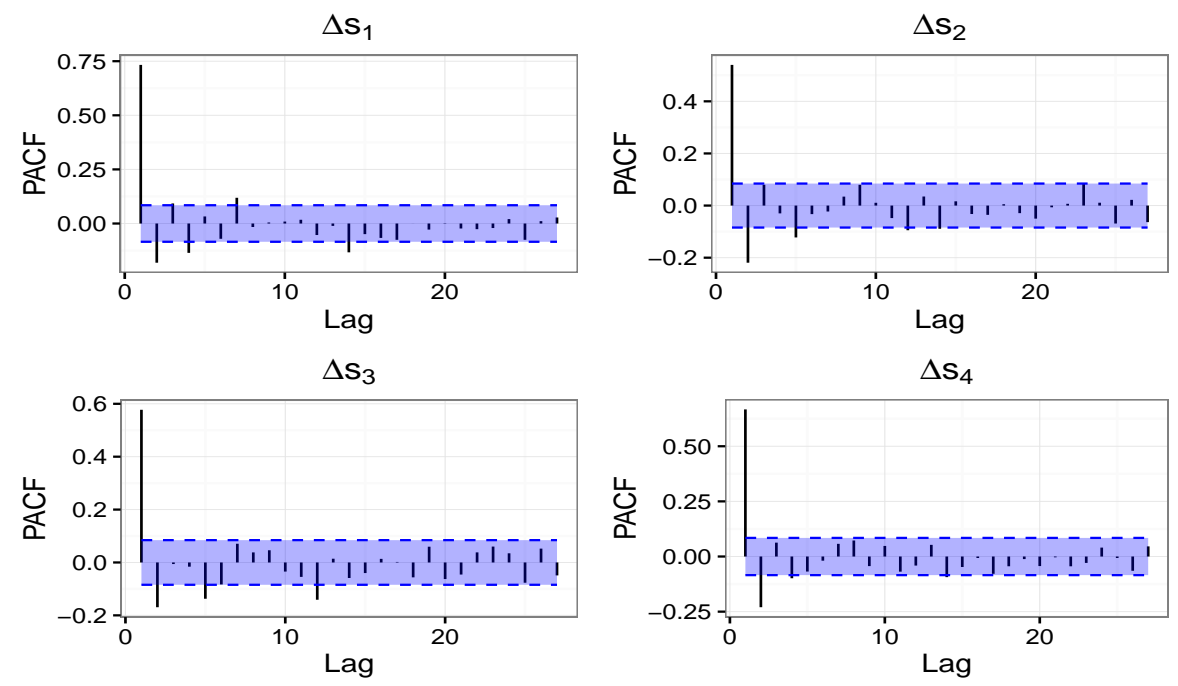

FIGURE 4. Partial autocorrelation plots for logarithmic freight rate data in first differences.

\begin{tabular}{cccc} 
& $\beta_{1}$ & $\beta_{2}$ & $\operatorname{std}(\epsilon)$ \\
\hline$\Delta s_{1}$ & 0.11 & -0.38 & 0.04 \\
$\Delta s_{2}$ & 0.29 & -0.38 & 0.08 \\
$\Delta s_{3}$ & 0.42 & -0.26 & 0.08 \\
$\Delta s_{4}$ & 0.48 & -0.36 & 0.06 \\
\hline
\end{tabular}

TABLE 3. Estimated parameters of the AR(2)-models

\begin{tabular}{lllll} 
& $\epsilon_{1}$ & $\epsilon_{2}$ & $\epsilon_{3}$ & $\epsilon_{4}$ \\
\hline$\epsilon_{1}$ & 1.0 & & & \\
$\epsilon_{2}$ & 0.48 & 1.0 & & \\
$\epsilon_{3}$ & 0.43 & 0.83 & 1.0 & \\
$\epsilon_{4}$ & 0.63 & 0.49 & 0.45 & 1.0 \\
\hline
\end{tabular}

TABLE 4. Lower diagonal of correlation matrix for estimated residuals of the fitted AR(2)-models

apart, correlation cannot help pull the series back together again. We can illustrate this in a small simulation experiment. For each of our four series we simulate future freight rates using the AR(2) models with parameters estimated above. The residuals are sampled from a multivariate normal distribution, with parameters for the correlation matrix given in Table 4 . Figure 5 shows the result from our simulation exercise. To the left of the vertical orange line is the historical data for the four routes, in total 540 observations. To the right we have our simulated series for the next 540 weeks. The strong positive correlation keeps the freight rates together for the start of the simulation period. But over time, the rates drift apart due to the randomness that is not common across routes. In the latter part of the simulation period, it is still easy to see that time series are positively correlated, but there is no gravity that pulls the time series together again. At the end of the simulation period, routes 3 and 4 end up close together by coincident, these two routes have the smallest correlation. Routes 1 and 2 have drifted far apart. This behavior will never happen in a competitive market, as ships would be re-routed to maximize profits, adjusting freight rates accordingly.

The economic reasoning suggesting that rates stick close together in the long run, resonates well with the statistical concept of cointegration. Let $\mathbf{u}_{t}=\left(u_{1, t},, u_{n, t}\right)^{\top}$ denote an $n$-dimensional vector of nonstationary time series, that needs to be differenced once to achieve stationarity. $\mathbf{u}_{t}$ is said to be cointegrated 


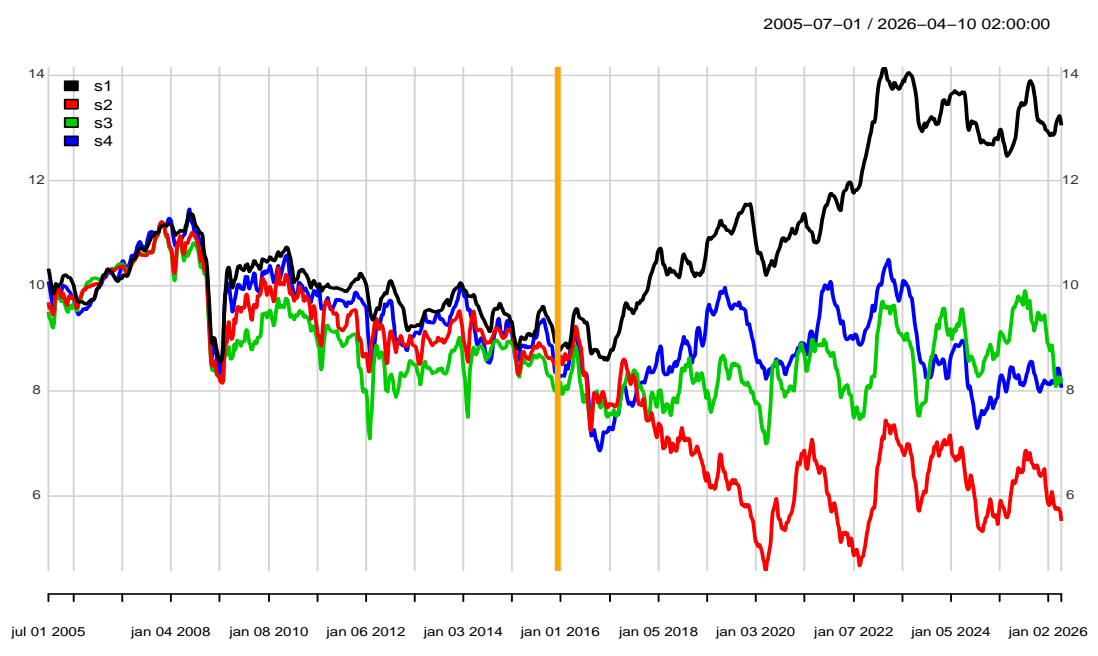

FIGURE 5. Historical and simulated correlated freight rate data. To the left of the vertical orange line is the historical data $(N=540)$. To the right of the vertical orange line is the simulated data $(N=540)$.

if there exists an $n$-dimensional vector $\mathbf{c}=\left(c_{1},, c_{n}\right)^{\top}$ such that

$$
\mathbf{c}^{\top} \mathbf{u}_{t}=\sum_{j=1}^{n} c_{j} u_{j, t},
$$

is stationary. If some coordinates of $\mathbf{c}$ are equal to zero then only the subset of time series in $\mathbf{u}_{t}$ with nonzero coefficients is cointegrated. If the $n$-dimensional vector time series is cointegrated with $0<r<n$ cointegrating vectors, then there are $n-r$ common stochastic trends (see e.g. Stock and Watson [45] for a discussion of the duality between cointegration vectors and common stochastic trends). If $r=0$, there is no cointegration. If $r=n-1$, there is a single common stochastic trend. In our case we expect the source of non-stationarity to be common to all rates to keep them all together in the long run, or, equivalently, $r=4-1=3$ cointegrating vectors.

Johansen [28] presents two test statistics for the number of cointegrating vectors within a vector autoregressive (VAR) modeling framework; the trace statistics and the maximum eigenvalue statistic. The trace statistic tests the null hypothesis: there are at most $r$ cointegrating relations against the alternative of $m$ cointegrating relations (i.e., the series are stationary), for $r=0,1, \ldots, m-1$. The maximum eigenvalue statistic tests the null hypohesis: there are $r$ cointegrating relations, against the alternative: there are $r+1$ cointegrating relations. In Table 5 we present the results from both tests based on a VAR(2) model. From the results in Table 5 we conclude that both tests indicate $r=3$ cointegrating relations vectors at a 5\% level of significance. In other words, the Johansen tests give statistical support to a single stochastic trend in the Supramax freight rate market. This is consistent with the maritime economic interpretation that overall supply/demand fundamentals govern a globally integrated freight market in the medium and long run, while the immediate regional supply/demand balance governs regional rates in the short run (Stopford [46])

So far our empirical investigations suggest persistent freight rates data that might appropriately be modeled using a single, common stochastic trend. In the next subsection we will propose a multivariate time series model consistent with these empirical facts.

3.1. A cointegrated time series model. In a freight market with $n$ regional freight rates, we assume there exists a common market factor with dynamics denoted by the time series $S_{t}$. We use the arithmetic average of the $n$ routes as the market factor, which in the case of Supramax coincides with the Baltic average tripcharter index underlying the FFA agreements. Setting $x_{t}=\ln S_{t}$ and recalling $s_{i, t}=\ln S_{i, t}$, we propose the following model for the logarithmic freight rate for each market segment $i=1, \ldots, n$ :

$$
s_{i, t}=x_{t}+y_{i, t} .
$$




\begin{tabular}{lllllllll}
\multicolumn{3}{c}{ Maximal eigenvalue test } & \multicolumn{5}{c}{ Trace test } \\
\hline & Statistic & $10 \%$ & $5 \%$ & $1 \%$ & Statistic & $10 \%$ & $5 \%$ & $1 \%$ \\
\hline$r \leq 3$ & 5.67 & 6.50 & 8.18 & 11.65 & 5.67 & 6.50 & 8.18 & 11.65 \\
$r \leq 2$ & $15.07^{*}$ & 12.91 & 14.90 & 19.19 & $20.75^{*}$ & 15.66 & 17.95 & 23.52 \\
$r \leq 1$ & $47.84 * *$ & 18.90 & 21.07 & 25.75 & $68.59^{* *}$ & 28.71 & 31.52 & 37.22 \\
$r \leq 0$ & $87.54 * *$ & 24.78 & 27.14 & 32.14 & $156.13^{* *}$ & 45.23 & 48.28 & 55.43 \\
\hline
\end{tabular}

TABLE 5. Johansen [28] tests for the number of cointegrating vectors for $n=4$ freight routes in the Supramax market.

\begin{tabular}{lrrrrr} 
& $x$ & $y_{1}$ & $y_{2}$ & $y_{3}$ & $y_{4}$ \\
\hline Mean & 9.7 & 0.3 & -0.1 & -0.5 & 0.1 \\
Median & 9.6 & 0.3 & -0.1 & -0.5 & 0.1 \\
Std. & 0.7 & 0.2 & 0.2 & 0.4 & 0.1 \\
Min & 8.3 & -0.2 & -0.8 & -1.7 & -0.4 \\
Max & 11.2 & 0.8 & 0.2 & 0.2 & 0.4 \\
\hline
\end{tabular}

TABLE 6. Descriptive statistics for common and regional-specific factors.

Each regional (logarithmic) spot rate can be decomposed into a common and a regional-specific factor. The regional-specific factors can be found by subtracting the common market factor from the regional spot rates, $y_{i, t}:=s_{i, t}-x_{t}$. Note that our market factor is observable from the $n$ market segments, making this decomposition straightforward to implement. The market factor and the regional-specific factors are all observable. Figure 6 shows the evolution of each factor, with the common market factor in the top panel, and all the regional-specific factors in the bottom panel. Summary statistics for $x_{t}$ and $y_{i, t}$ are given in
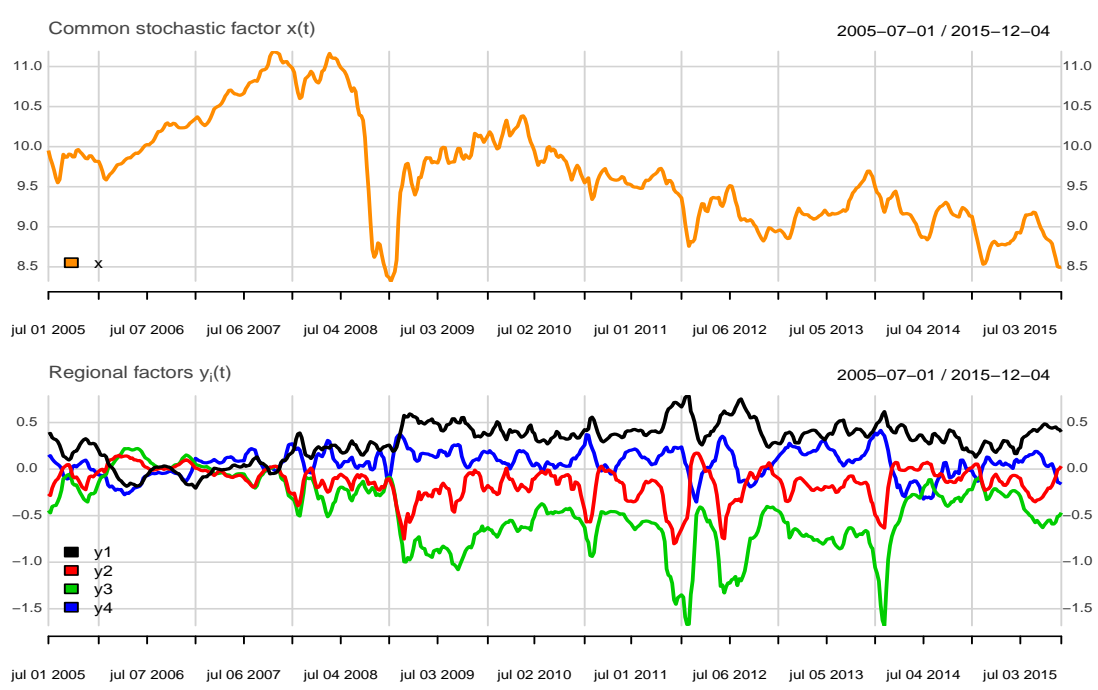

FIGURE 6. Freight rate decomposition into a common market factor and different regional-specific factors.

Table 6. From the vertical axes in Figure 6 and the descriptive statistics in table 6 it is clear that the common factor picks up most of the variation in the freight rates. The regional factors represent minor adjustments to the overall market factor, consistent with the observation that short-term regional over- or undersupply of ships can lead to temporary regional differences in spot rates. The regional factors are negative, on average, for route 2 and route 3 , and positive for route 1 and route 4 . As routes 1 and 4 originate in the Atlantic 
basin, and routes 2 and 3 originate in the Pacific basin, this difference simply reflects the well-known Atlantic premium in drybulk freight rates (see, Adland et al. [1], 2017, for a thorough discussion). Just inspecting Figure 6, it seems that route 1 and route 4 are positively correlated, and so is route 2 and route 3. This is also expected, and reflects the fact that ships open in the Atlantic competes for cargoes on routes 1 and 4, while ships open in the Pacific have a choice between routes 2 and 3, respectively. This "joint" supply will impose some positive correlation. Furthermore, route 1 and route 4 seem to be negatively correlated with route 2 and route 3 . We expect that this slightly asynchronous development in the Atlantic and Pacific freight rates reflects a degree of "overshooting" in the movement of tonnage between the two key regions. Unlike the common factor, the regional factors all seem to have mean reverting features. Next, we perform unit root tests on the common and regional factors, before specifying an appropriate dynamic factor model.

We perform the same unit root tests as we did for the regional freight rates above. The results are presented in Table 7. Both the ADF-GLS and PT tests agree that the null hypothesis of non-stationary regional factors can be rejected in favor of the stationary alternative. Turning to the common factor, both the ADF-GLS test and the PT test accept the null hypothesis of non-stationarity. After differencing the data once, the tests reject the null hypothesis of non-stationarity at $5 \%$ level of significance.

\begin{tabular}{lcccccc}
\multicolumn{4}{c}{ Regional-specific factors } & \multicolumn{3}{c}{ Common factor } \\
\hline & $y_{1}$ & $y_{2}$ & $y_{3}$ & $y_{4}$ & $x$ & $\Delta x$ \\
\hline ADF-GLS & $-3.29^{* *}$ & $-5.42^{* *}$ & $-3.76^{* *}$ & $-5.56^{* *}$ & -1.59 & $-2.85^{* *}$ \\
PT & $1.14^{* *}$ & $0.53^{* *}$ & $0.86^{* *}$ & $0.56^{* *}$ & 4.44 & $2.14^{*}$ \\
Lags & 1 & 1 & 1 & 1 & 4 & 7 \\
\hline
\end{tabular}

TABLE 7. Unit root tests for the regional-specific factors $y_{i}$, the common factor $x$ and the common factor in first differences $\Delta x$. Critical levels for the ADF-GLS test on $1 \%$ and $5 \%$ level of significance is -2.58 and -1.98 , respectively. Critical levels for PT test on $1 \%$ and $5 \%$ level of significance is 1.78 and 3.17 , respectively. The BIC criterion is used lag length selection.

Overall, this suggests that the common factor is non-stationary (the common stochastic trend), while the regional-specific factors are stationary mean reverting processes around this trend. Next we estimate dynamic models for both the market factor and the regional factors.

We assume that the market factor $x_{t}$ can be modeled as an autoregressive integrated moving average (ARIMA) process. We have already established that this is a non-stationary process that needs to be differenced once to achieve stationarity. We use the BIC criterion to determine the number of lags, which result in an $\mathrm{AR}(4)$-process without constant term for $\Delta x_{t}$, with the following parameters:

$$
\Delta x_{t}=0.93 \Delta x_{t-1}-0.48 \Delta x_{t-2}+0.28 \Delta x_{t-3}-0.13 \Delta x_{t-4}+\epsilon_{x, t}, \quad \operatorname{std}\left(\epsilon_{x, t}\right)=0.05 .
$$

We assume that the regional-specific factors can be modeled by a higher order autoregressive model. Once more we use the BIC criterion for lag selection. This results in an AR(2)-model with significant constant terms for all regional factors,

$$
y_{i, t}=\mu_{i}+\beta_{1, i} y_{i, t-1}+\beta_{2, i} y_{i, t-2}+\epsilon_{i, t}, i=1,2,3,4,
$$

with parameter estimates presented in Table 8 . Correlations between estimated residuals are given in Ta-

\begin{tabular}{c|cccc} 
& $\mu$ & $\beta_{1}$ & $\beta_{2}$ & $\operatorname{std}(\epsilon)$ \\
\hline$y_{1}$ & 0.3 & 1.51 & -0.53 & 0.03 \\
$y_{2}$ & -0.14 & 1.46 & -0.54 & 0.04 \\
$y_{3}$ & -0.46 & 1.55 & -0.57 & 0.05 \\
$y_{4}$ & 0.0 & 1.5 & -0.55 & 0.03 \\
\hline
\end{tabular}

TABLE 8. Estimated parameters for the regional AR(2) models

ble 9 . We see that the estimated residuals for the regional-specific factors for route 2,3 and 4 are positively 
correlated with the changes in the market factor $x_{t}$ (the latter two only weakly positively correlated). For route 1 the corresponding correlation is negative.

\begin{tabular}{r|rrrrr} 
& $\epsilon_{x}$ & $\epsilon_{1}$ & $\epsilon_{2}$ & $\epsilon_{3}$ & $\epsilon_{4}$ \\
\hline$\epsilon_{x}$ & 1.0 & & & & \\
$\epsilon_{1}$ & -0.4 & 1.0 & & & \\
$\epsilon_{2}$ & 0.3 & -0.7 & 1.0 & & \\
$\epsilon_{3}$ & 0.2 & -0.6 & 0.6 & 1.0 & \\
$\epsilon_{4}$ & 0.1 & -0.1 & -0.5 & -0.5 & 1.0
\end{tabular}

TABLE 9. Lower diagonal of correlation matrix for estimated residuals

Correlation between estimated residuals for the regional factors shows the pairwise pattern observed already from the time series plot in Figure 6. Route 2 and route 3 are positively correlated with estimated coefficient of correlation of 0.6 . Correspondingly, route 1 is negatively correlated with all other routes, route 2 is negatively correlated with route 4 and the same between routes 3 and 4 .

As a validation of our model, we build a simulation engine for regional freight rates to see graphically how it performs. Now the modeling process is reversed. We proceed in three steps:

(1) Draw correlated random error terms for $\epsilon_{x, t}$ and $\epsilon_{i, t}$ from the multivariate normal distribution using the correlation matrix in Table 9 as input.

(2) Simulate univariate $\operatorname{AR}(p)$ models for $\Delta x_{t}$ and $y_{i, t}$ using the estimated models from (16) and Table 8.

(3) Compute the logarithmic regional freight rates using (15).

Figure 7 shows a single simulation of our model that compares directly to the case of correlated regional freight rates in Figure 5.

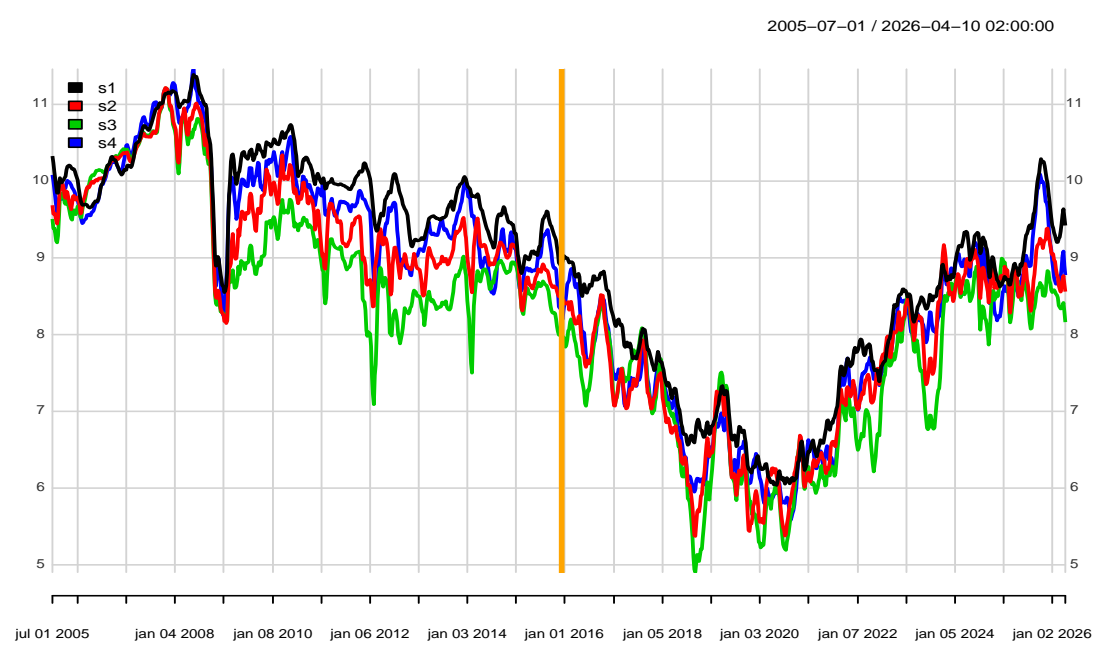

FIGURE 7. Historical and simulated freight rate data based on cointegration model. To the left of the vertical line is the historical data $(N=540)$. To the right of the vertical line is the simulated data $(N=540)$.

We note that our cointegrated time series model can be viewed as a discrete-time analogue of the continuous-time framework proposed in Benth and Koekebakker [15]. There the authors propose a factor model for cointegrated commodity prices by splitting into a non-stationary common factor (a stochastic trend) and stationary specific factors, based on drifted Brownian motion and continuous-time autoregressive moving average processes, respectively. One of the main messages of Benth and Koekebakker [15] is that cointegration in the spot market is inherited by the forward market, when one considers fixed time to 
maturities. This is true in commodity markets which are incomplete, that is, where there are market frictions preventing the buy and hold strategy to be used in replicating forward contracts. The freight market constitutes a prime example of such an incomplete market as the transportation service cannot be stored or traded. In the next section we will analyse forward freight rate agreements for our cointegrated model.

3.2. Calibrating the continuous-time model to data. We apply the data analysis and time series model to assess the parameters of the continuous-time dynamics (10) proposed in the previous section.

First, we consider a market with $n=4$ routes, and recall that the regional-specific factor time series where found to be $\mathrm{AR}(2)$ processes with a level. Hence, we assume that $Y_{i}, i=1,2,3,4$ to be modelled by $\mathrm{CAR}(2)$-processes, which means that $p_{i}=2, i=1,2,3,4$. In our definition of a CAR $(p)$-process, we have assumed a zero level. To correct for this, we make the following slight change in the definition of logarithmic spot freight prices, that is, we assume that $\bar{Y}_{i}:=Y_{i}+\theta_{i}$ and define $\ln S_{i}$ to have the dynamics

$$
\ln S_{i}(t)=X(t)+\bar{Y}_{i}(t)
$$

for $i=1,2,3,4$.. The parameters $\theta_{i}, i=1,2,3,4$ are all assumed to be constants.

From Proposition 1 we have that a CAR(2)-process $\{Y(t)\}_{t \geq 0}$ with volatility $\sigma$ and CAR-matrix $A$ as in (2) for $p=2$ can be expressed on a discrete time scale $t=0,1,2, \ldots$, with time stepping $\delta=1$ as the $\operatorname{AR}(2)$-process $\left\{y_{t}\right\}_{t=0,1,2 \ldots}$,

$$
y_{t}=\left(2-\alpha_{1}\right) y_{t-1}+\left(\alpha_{1}-\alpha_{2}-1\right) y_{t-2}+\sigma \epsilon_{t} .
$$

Here, $\left\{\epsilon_{t}\right\}_{t=0,1, \ldots}$ is a time series of IID standard normally distributed random variables (white noise). But then $\bar{Y}(t):=Y(t)+\theta$ for a constant $\theta$ has the time series representation,

$$
\bar{y}_{t}=\theta \alpha_{2}+\left(2-\alpha_{1}\right) \bar{y}_{t-1}+\left(\alpha_{1}-\alpha_{2}-1\right) \bar{y}_{t-2}+\sigma \epsilon_{t} .
$$

We compare the estimated parameters in Table (8) with (19) in order to find $\alpha_{1}, \alpha_{2}, \theta$ and $\sigma$ for each of the four regional-specific factor processes $\left\{\bar{Y}_{i}(t)\right\}_{t \geq 0}$. The results are shown in Table 10. We may ask

\begin{tabular}{ccccc} 
& $\alpha_{1}$ & $\alpha_{2}$ & $\theta$ & $\sigma$ \\
\hline $\bar{Y}_{1}$ & 0.49 & 0.02 & 15 & 0.03 \\
$\bar{Y}_{2}$ & 0.54 & 0.08 & -1.75 & 0.04 \\
$\bar{Y}_{3}$ & 0.45 & 0.02 & -23 & 0.05 \\
$\bar{Y}_{4}$ & 0.50 & 0.05 & 0 & 0.03 \\
\hline
\end{tabular}

TABLE 10. Calibrated CAR(2)-parameters for the regional-specific continuous-time model.

whether the four CAR-matrices defined from the estimated $\alpha$ 's in Table 10 have eigenvalues with negative real part to ensure a stationary dynamics. Indeed, as we see in Table 11, this is true, and we can conclude that $\bar{Y}_{i}, i=1,2,3,4$ all have a stationary normal distribution in the limit as time tends to infinity.

\begin{tabular}{rrr} 
& $\lambda_{1}$ & $\lambda_{2}$ \\
\hline $\bar{Y}_{1}$ & -0.045 & -0.45 \\
$\bar{Y}_{2}$ & $-0.27+0.08 \mathrm{i}$ & $-0.27-0.08 \mathrm{i}$ \\
$\bar{Y}_{3}$ & -0.050 & -0.40 \\
$\bar{Y}_{4}$ & -0.14 & -0.36 \\
\hline
\end{tabular}

TABLE 11. Eigenvalues for the CAR-matrices from the calibrated CAR(2)-parameters in Table 10 for the regional-specific continuous-time model.

By Proposition 4, the common factor $X$ is suggested to be an integrated $\operatorname{AR}\left(p_{0}\right)$ model on a discrete scale. From (16), we find $p_{0}=4$, and using this autoregressive order in Proposition 4 yields the time series dynamics

$$
\begin{aligned}
\Delta x_{t}=( & \left.-\alpha_{1}\right) \Delta x_{t-1}+\left(3 \alpha_{1}-\alpha_{2}-6\right) \Delta x_{t-2} \\
& +\left(2 \alpha_{2}-3 \alpha_{1}-\alpha_{3}+4\right) \Delta x_{t-3}+\left(\alpha_{1}-\alpha_{2}+\alpha_{3}-\alpha_{4}-1\right) \Delta x_{t-4}+\sigma_{0} \epsilon_{t}
\end{aligned}
$$


where $\alpha_{1}, \ldots, \alpha_{4}$ are the four parameters in the CAR-matrix $A_{0}$ of the CAR(4)-process $Y_{0}$ and $\left\{\epsilon_{t}\right\}_{t=0,1,2 \ldots}$. are IID normally distributed random variables. We identify from (16)

$$
\alpha_{1}=3.07, \quad \alpha_{2}=3.69, \quad \alpha_{3}=1.89, \quad \alpha_{4}=0.58 .
$$

The estimated volatility becomes $\sigma_{0}=0.05$. These estimates yield a CAR-matrix $A_{0}$ for $X$ which has eigenvalues $\lambda_{1,2}=-1.237 \pm 0.6181 \mathrm{i}$ and $\lambda_{3,4}=-0.298 \pm 0.4632 \mathrm{i}$, i.e., all four eigenvalues have negative real part. We conclude that $Y_{0}$ is a stationary $\operatorname{CAR}(4)$ process, while $X=\int_{0}^{t} Y_{0}(s) d s$ is a non-stationary process.

We model the noise process $\mathbf{B}$ as a 5-dimensional Brownian motion, correlated according to the matrix of correlation coefficients found in Table 9. I.e., we suppose that $\operatorname{corr}\left(B_{i}(t), B_{j}(t)\right)=\rho_{i, j} t$ for $i, j=$ $0,1,2,3,4$, where $\rho_{i, j}$ are the coefficients in the $5 \times 5$ matrix,

$$
\Gamma=\left[\begin{array}{rrrrr}
1.0 & -0.4 & 0.3 & 0.2 & 0.1 \\
-0.4 & 1.0 & -0.7 & -0.6 & -0.1 \\
0.3 & -0.7 & 1.0 & 0.6 & -0.5 \\
0.2 & -0.6 & 0.6 & 1.0 & -0.5 \\
0.1 & -0.1 & -0.5 & -0.5 & 1.0
\end{array}\right]
$$

This is a positive definite matrix, as all the eigenvalues are positive, and thus a valid correlation matrix for the Brownian motions. Hence, we have fully specified a cointegrated model in continuous-time for the freight market we study.

\section{FFA PRICING}

We suppose that there are available FFA written on the common factor, in the sense that there are contracts delivering the common market factor at time $T$. In our modelling context, this means that one can trade in FFA's with financial delivery of

$$
S(T)=\exp (X(T)),
$$

at time $T$. Following the arguments of Benth, Šaltytė Benth and Koekebakker [13, Sect. 4.1] for nonhedgeable forwards, we define the FFA price $F_{X}(t, T)$ at time $t \leq T$ for a contract delivering the common factor $S(T)$ at time $T>0$ as

$$
F_{X}(t, T)=\mathbb{E}_{Q}\left[S(T) \mid \mathcal{F}_{t}\right],
$$

where $Q \sim P$ is a pricing measure. Note that the probability $Q$ is not necessarily a risk-neutral probability in the sense that the discounted common factor process $t \mapsto e^{-r t} S(t)$ is a $Q$-martingale, with $r>0$ being the risk-free interest rate. The pricing measure $Q$ models the risk premium in the FFA market, and here we will assume that it takes the simple form of a constant market price of risk $\gamma_{0} \in \mathbb{R}$ defined as follows: Introduce the stochastic process

$$
d W_{0}(t)=\gamma_{0} d t+d B_{0}(t) .
$$

Then, by Girsanov's Theorem (see Karatzas and Shreve [29, Thm. 5.1, Ch. 3]), there exists a probability $Q \sim P$ such that $W_{0}$ is a $Q$-Brownian motion. We will use this $Q$ as our pricing measure, and we observe that the $Q$-dynamics of $Y_{0}$ in the definition of the common market factor process $X$ becomes $Y_{0}(t)=\mathbf{e}_{1}^{\top} \mathbf{Z}_{0}(t)$ where from (1),

$$
d \mathbf{Z}_{0}(t)=\left(A_{0} \mathbf{Z}_{0}(t)-\sigma_{0} \gamma_{0} \mathbf{e}_{p_{0}}\right) d t+\sigma_{0} \mathbf{e}_{p_{0}} d W_{0}(t) .
$$

We remark in passing that while the CAR-matrix and $\sigma_{0}$ can be estimated from market prices as we showed in Section 2, the market price of risk $\gamma_{0}$ must be calibrated from observed FFA prices. Note also that we have assumed a rather restrictive class of parametric pricing measures here. Indeed, following Benth and Šaltyte Benth [12, Proposition 5.1] (or also the discussion in Benth and Koekebakker [15]), one can introduce pricing measures $Q$ that not only changes the level of $\mathbf{Z}_{0}$ by some parameter $\gamma_{0}$, but also changes the $\alpha$ 's in the CAR-matrix. We refrain from such generality here, as we may always simply re-interpret the CAR-matrix.

In the next Proposition we derive the FFA price (the proof is found in Appendix A). 
Proposition 5. Suppose the common factor $X$ is defined as in (11). Then for every $0 \leq t \leq T$ we have

$$
F_{X}(t, T)=S(t) \exp \left(h\left(\mathbf{Z}_{0}(t), T-t\right)\right),
$$

where

$$
\begin{aligned}
h(\mathbf{z}, s)=\mathbf{e}_{1}^{\top} & A_{0}^{-1}\left(e^{A_{0} s}-I_{p_{0}}\right) \mathbf{z}-\sigma_{0} \gamma_{0} \mathbf{e}_{1}^{\top} A_{0}^{-2}\left(e^{A_{0} s}-I_{p_{0}}\right) \mathbf{e}_{p_{0}} \\
& +\sigma_{0} \gamma_{0} \mathbf{e}_{1}^{\top} A_{0}^{-1} \mathbf{e}_{p_{0}} s+\frac{\sigma_{0}^{2}}{2} \int_{0}^{s} \mathbf{e}_{p_{0}}^{\top}\left(e^{A_{0}^{\top} u}-I_{p_{0}}\right) A_{0}^{-\top} \mathbf{e}_{1} \mathbf{e}_{1}^{\top} A_{0}^{-1}\left(e^{A_{0} u}-I_{p_{0}}\right) \mathbf{e}_{p_{0}} d u
\end{aligned}
$$

for $s \geq 0$ and $\mathbf{z} \in \mathbb{R}^{p_{0}}$.

The inverse of a CAR-matrix $A$ is explicitly available (see Lemma 4.2 in Benth and Šaltytė Benth [12]). Hence, we have an explicit expression for $A_{0}^{-1}$ in Proposition 5 above.

Indeed, as the next result shows, the FFA dynamics is a geometric Brownian motion with time-dependent volatility (the proof is relegated to Appendix A):

Proposition 6. The FFA price $F_{X}(t, T)$ has the $Q$-dynamics

$$
\frac{d F_{X}(t, T)}{F_{X}(t, T)}=\sigma_{0}\left(\mathbf{e}_{1}^{\top} A_{0}^{-1}\left(e^{A_{0}(T-t)}-I_{p_{0}}\right) \mathbf{e}_{p_{0}}\right) d W_{0}(t) .
$$

As a direct consequence of this proposition we find the market dynamics of $F_{X}$ to be

$$
\frac{d F_{X}(t, T)}{F_{X}(t, T)}=\sigma_{0} \gamma_{0}\left(\mathbf{e}_{1}^{\top} A_{0}^{-1}\left(e^{A_{0}(T-t)}-I_{p_{0}}\right) \mathbf{e}_{p_{0}}\right) d t+\sigma_{0}\left(\mathbf{e}_{1}^{\top} A_{0}^{-1}\left(e^{A_{0}(T-t)}-I_{p_{0}}\right) \mathbf{e}_{p_{0}}\right) d B_{0}(t) .
$$

This shows that $\gamma_{0}$ has a natural intepretation as the market price of risk. Concerning the volatility term structure $\Sigma_{X}(T-t), T \geq t$, of $F_{X}$, where

$$
\Sigma_{X}(s)=\sigma_{0}\left(\mathbf{e}_{1}^{\top} A_{0}^{-1}\left(e^{A_{0} s}-I_{p_{0}}\right) \mathbf{e}_{p_{0}}\right)
$$

for $s \geq 0$, we have:

Corollary 7. Assume the CAR-matrix of $Y_{0}$ have eigenvalues with negative real part. The volatility term structure $\Sigma_{X}(s)$ defined in (24) for $s \geq 0$ satisfies $\Sigma_{X}(0)=0$ and $\lim _{s \rightarrow \infty} \Sigma_{X}(s)=\sigma_{0} / \alpha_{p_{0}}$.

The proof of this result is found in Appendix A. We note that in the long end of the FFA market, the volatility is constant $\sigma_{0} / \alpha_{p_{0}}$. In the specific case of the market we consider in this paper, we recall from Sections 2 and 3 that $p_{0}=4, \alpha_{4}=0.58$ and $\sigma_{0}=0.05$, and hence the long term volatility is estimated to be 0.086 , or $62 \%$ annually. ${ }^{2}$ In Figure 8 we plot the volatility term structure $\Sigma_{X}$ (solid curve) as a function of time to maturity (measured in weeks) for the set of estimated parameters found in Section 3 . The volatility is positive and converges asymptotically to the long term level of $62 \%$ for FFA contracts delivering in around half a year. The maximum volatility is just below 70\%, and is reached for the FFA with delivery in 8 weeks. Interestingly, the volatility is an increasing function in time to maturity, from zero up to its maximum reached at 8 weeks time to maturity. Next, it has a bump with a minimum at around 15 weeks to maturity. After a slight increase again, it stabilizes. This complex term structure is a reflection of the memory inherited from the CAR(4)-process $Y_{0}$ and the integrating feature of $X$.

In the market there exist no exchange-traded FFA's on the regional routes. However, we still compute the FFA prices on each route, which is readily available from our theoretical framework. To this end, let $\gamma \in \mathbb{R}^{n+1}$ be the vector $\gamma=\left(\gamma_{0}, \gamma_{1}, \ldots, \gamma_{n}\right)^{\top}$, and from Girsanov's Theorem (see Karatzas and Shreve [29, Thm 5.1, Ch. 3]), there exists a probability $Q \sim P$ such that $\mathbf{W}$ defined as

$$
d \mathbf{W}(t)=\gamma d t+d \mathbf{B}(t)
$$

is a $Q$-Brownian motion, where the correlation structure from $\mathbf{B}$ is inherited. Thus, we extend the pricing probability $Q$ introduced for the market factor $X$ above to also include a risk premium $\gamma_{i}, i=1, \ldots, n$ for each of the $n$ regional routes. It follows immediately from (1) that for $Y_{i}(t)=\mathbf{e}_{1}^{\top} \mathbf{Z}_{i}(t)$, the $Q$-dynamics of $\mathbf{Z}_{i}(t) \in \mathbb{R}^{p_{i}}$ is given by

$$
d \mathbf{Z}_{i}(t)=\left(A_{i} \mathbf{Z}_{i}(t)-\sigma_{i} \gamma_{i} \mathbf{e}_{p_{i}}\right) d t+\sigma_{i} \mathbf{e}_{p_{i}} d W_{i}(t),
$$

\footnotetext{
${ }^{2}$ We assume 52 trading weeks in this calculation.
} 
for $i=1, \ldots, n$. We assume that the FFA price at time $t \geq 0$ for delivery of the regional route $i, i=$ $1, \ldots, n$ at time $T \geq t$, denoted $F_{i}(t, T)$, is given by

$$
F_{i}(t, T)=\mathbb{E}_{Q}\left[S_{i}(T) \mid \mathcal{F}_{t}\right]
$$

with $S_{i}(t)$ given by (10). We compute the price to obtain the following:

Proposition 8. Suppose that the common factor $X$ is defined as in (11) and the regional-specific factor $Y_{i}$ is a $\operatorname{CAR}\left(p_{i}\right)$-process. Then for every $0 \leq t \leq T$ and $i=1, \ldots, n$, we have

$$
F_{i}(t, T)=S(t) \exp \left(h\left(\mathbf{Z}_{0}(t), T-t\right)+h_{i}\left(\mathbf{Z}_{i}(t), T-t\right)\right)
$$

where $h\left(\mathbf{z}_{0}, s\right)$ is defined in Proposition 5 and

$$
\begin{aligned}
h_{i}\left(\mathbf{z}_{i}, s\right) & =\mathbf{e}_{1}^{\top} e^{A_{i} s} \mathbf{z}_{i}-\sigma_{i} \gamma_{i} \mathbf{e}_{1}^{\top} A_{i}^{-1}\left(e^{A_{i} s}-I_{p_{i}}\right) \mathbf{e}_{p_{i}}+\frac{1}{2} \int_{0}^{s} \sigma_{i}^{2}\left(\mathbf{e}_{1}^{\top} e^{A_{i} u} \mathbf{e}_{p_{i}}\right)^{2} d u \\
& +\int_{0}^{s} \rho_{0, i} \sigma_{0} \sigma_{i}\left(\mathbf{e}_{1}^{\top} A_{0}^{-1}\left(e^{A_{0} u}-I_{p_{0}}\right) \mathbf{e}_{p_{0}}\right)\left(\mathbf{e}_{1}^{\top} e^{A_{i} u} \mathbf{e}_{p_{i}}\right) d u
\end{aligned}
$$

for $\mathbf{z}_{0} \in \mathbb{R}^{p_{0}}, \mathbf{z}_{i} \in \mathbb{R}^{p_{i}}$ and $s \geq 0$ and $\rho_{0, i}$ being the correlation coefficient between $W_{0}$ and $W_{i}$.

An alternative expression for $F_{i}(t ; T)$ is

$$
F_{i}(t, T)=F_{X}(t, T) \exp \left(h_{i}\left(\mathbf{Z}_{i}(t), T-t\right)\right),
$$

with $h_{i}$ defined in Proposition 8. We can also show for the regional FFA that its dynamics is a geometric Brownian motion with a time-dependent volatility;

Proposition 9. The FFA price $F_{i}(t, T)$ has the $Q$-dynamics

$$
\frac{d F_{i}(t, T)}{F_{i}(t, T)}=\sigma_{0} \mathbf{e}_{1}^{\top} A_{0}^{-1}\left(e^{A_{0}(T-t)}-I_{p_{0}}\right) \mathbf{e}_{p_{0}} d W_{0}(t)+\sigma_{i} \mathbf{e}_{1}^{\top} e^{A_{i}(T-t)} \mathbf{e}_{p_{i}} d W_{i}(t)
$$

Now, introducing the notation

$$
\Sigma_{Y_{i}}(s)=\sigma_{i} \mathbf{e}_{1}^{\top} e^{A_{i} s} \mathbf{e}_{p_{i}}
$$

for $s \geq 0$, we see that the volatility term structure of the FFA for route $i$ is defined by

$$
\Sigma_{i}^{2}(s)=\Sigma_{X}^{2}(s)+2 \rho_{0, i} \Sigma_{X}(s) \Sigma_{Y_{i}}(s)+\Sigma_{Y_{i}}^{2}(s) .
$$

In Figure 8 we have plotted the volatility term structure of route 1 (broken curve, left graphics), where we
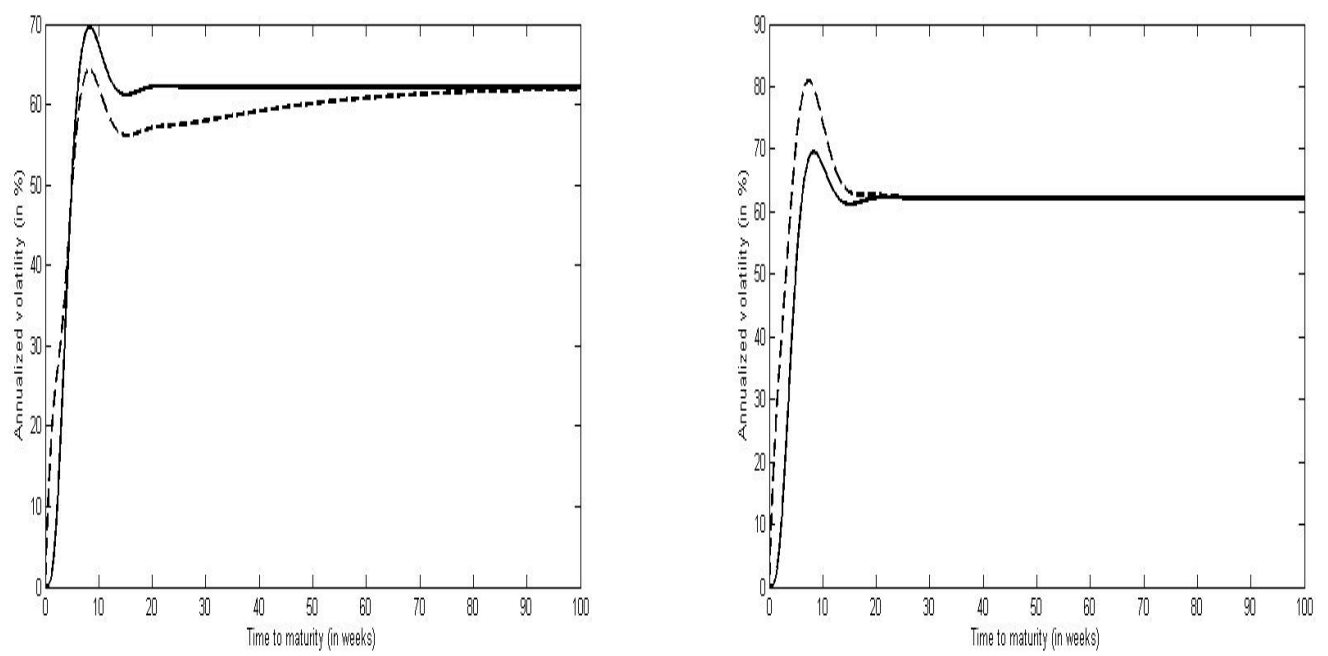

FIGURE 8. Plot of the volatility term structures $\Sigma_{1}(s)$ (broken curve, left) and $\Sigma_{2}(s)$ (broken curve, right) defined in (30) for the estimated parameters from Section 3, along with the $\Sigma_{X}$ (solid curve). 
recall the parameters from Section 3 and the correlation coefficient $\rho_{0,1}=-0.4$. There are some deviations from the common factor term structure volatility, which is shown as a solid curve. The regional volatility is slightly above, until approximately 5 weeks out on the FFA curve where the regional volatility goes below but follows the shape of the common factor volatility. The regional volatility converges asymptotically to the long term level, which is slightly above $62 \%$. Indeed, since in in general

$$
\lim _{s \rightarrow \infty} \Sigma_{Y_{i}}(s)=0
$$

when $A_{i}$ has eigenvalues with negative real part, we see that

$$
\lim _{s \rightarrow \infty} \Sigma_{i}(s)=\lim _{s \rightarrow \infty} \Sigma_{X}(s)=\sigma_{0} / \alpha_{p_{0}}
$$

whenever $A_{0}$ has eigenvalues with negative real part. For route 1 , the correlation with the market common factor was negative. The same example for route 2 yields the volatility term structure as depicted in Figure 8 (broken curve, right graphics). For this positively correlated case, the difference between the market common factor and the route becomes more pronounced, as well as always yielding a higher volatility for all maturities. Indeed, the maximal volatility for route 2 is $81 \%$.

We end this section with a discussion of the correlation term structure between route $i$ and the common market factor. Define the correlation term structure by the function $s \mapsto R_{i}(s), s \geq 0$, where

$$
R_{i}(T-t)=\frac{\operatorname{Cov}\left(d F_{X}(t, T) / F_{X}(t, T), d F_{i}(t, T) / F_{i}(t, T)\right)}{\sqrt{\operatorname{Var}\left(d F_{X}(t, T) / F_{X}(t, T)\right)} \sqrt{\operatorname{Var}\left(d F_{i}(t, T) / F_{i}(t, T)\right.}} .
$$

Seemingly $R_{i}$ will depend on $t$ and $T$, however, we find

$$
\begin{gathered}
\operatorname{Cov}\left(d F_{X}(t, T) / F_{X}(t, T), d F_{i}(t, T) / F_{i}(t, T)\right)=\left(\Sigma_{X}^{2}(T-t)+\rho_{0, i} \Sigma_{X}(T-t) \Sigma_{Y_{i}}(T-t)\right) d t, \\
\operatorname{Var}\left(d F_{X}(t, T) / F_{X}(t, T)\right)=\Sigma_{X}^{2}(T-t) d t
\end{gathered}
$$

and

$$
\operatorname{Var}\left(d F_{i}(t, T) / F_{i}(t, T)\right)=\left(\Sigma_{X}^{2}(T-t)+2 \rho_{0, i} \Sigma_{X}(T-t) \Sigma_{Y_{i}}(T-t)+\Sigma_{Y_{i}}^{2}(T-t)\right) d t
$$

We have plotted the correlation term structure in Figure 9, for route 1 and route 2 respectively. The shape for route 1 is rather peculiar.
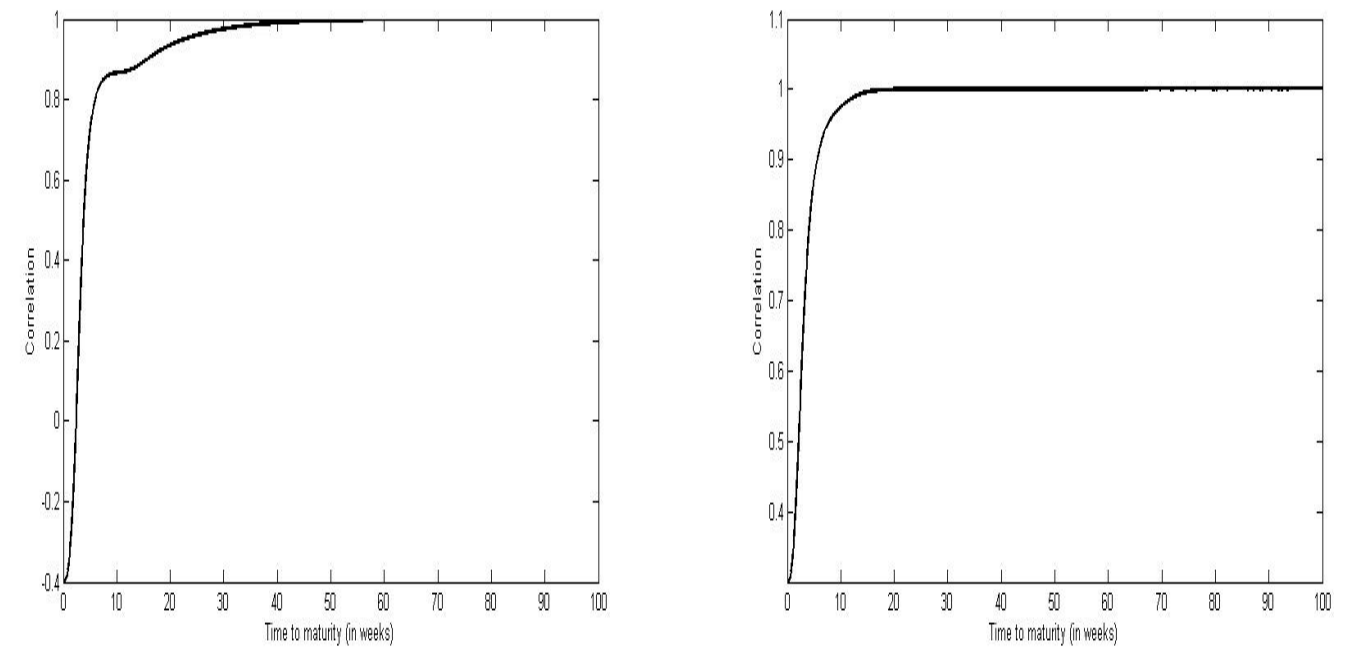

FIGURE 9. Plot of the correlation term structure between route 1 and the common factor (left), and route 2 and the common factor (right), for the estimated parameters in Section 2. 
4.1. Dynamics of FFA's with settlement period. Admittedly, the FFA's traded in the market are settled over a period and not instantaneously. These periods are typically calendar months. Assume the FFA is settled over the period $\left[T_{1}, T_{2}\right], T_{1}<T_{2}$, and denote the FFA price $\bar{F}\left(t, T_{1}, T_{2}\right), t \leq T_{1}$. From Benth et al. [13], a no-arbitrage relationship between $\bar{F}\left(t, T_{1}, T_{2}\right)$ and $F(t, T)$ is

$$
\bar{F}\left(t, T_{1}, T_{2}\right)=\frac{1}{T_{2}-T_{1}} \int_{T_{1}}^{T_{2}} F(t, T) d T,
$$

as the market settles on the average spot rate over the settlement period. We have used $F$ and $\bar{F}$ as a generic notation here.

From the geometric Brownian motion dynamics of $F_{X}$ and $F_{i}$ in Props. 6 and 9 with maturity-dependent volatility, it seems impossible to derive a reasonable closed-form expression for the dynamics of $t \mapsto$ $\bar{F}_{X}\left(t, T_{1}, T_{2}\right)$ and $t \mapsto \bar{F}_{i}\left(t, T_{1}, T_{2}\right)$. Here we propose an approximation, where we preserve the geometric Brownian motion dynamics but average the volatility term structure over the settlement period, i.e., we suppose that

$$
\frac{d \bar{F}_{X}\left(t, T_{1}, T_{2}\right)}{\bar{F}_{X}\left(t, T_{1}, T_{2}\right)}=\bar{\Sigma}_{X}\left(t, T_{1}, T_{2}\right) d W_{0}(t)
$$

with

$$
\bar{\Sigma}_{X}\left(t, T_{1}, T_{2}\right):=\frac{1}{T_{2}-T_{1}} \int_{T_{1}}^{T_{2}} \Sigma_{X}(T-t) d T, t \leq T_{1}
$$

and for $i=1, \ldots, n$,

$$
\frac{d \bar{F}_{i}\left(t, T_{1}, T_{2}\right)}{\bar{F}_{i}\left(t, T_{1}, T_{2}\right)}=\bar{\Sigma}_{X}\left(t, T_{1}, T_{2}\right) d W_{0}(t)+\bar{\Sigma}_{Y_{i}}\left(t, T_{1}, T_{2}\right) d W_{i}(t)
$$

with

$$
\bar{\Sigma}_{Y_{i}}\left(t, T_{1}, T_{2}\right):=\frac{1}{T_{2}-T_{1}} \int_{T_{1}}^{T_{2}} \Sigma_{Y_{i}}(T-t) d T, t \leq T_{1} .
$$

We recall $\Sigma_{X}(s)$ and $\Sigma_{Y_{i}}(s)$ from (24) and (29), respectively. We find:

Proposition 10. For $t \leq T_{1}<T_{2}$, it holds that

$$
\bar{\Sigma}_{X}\left(t, T_{1}, T_{2}\right)=\sigma_{0} \mathbf{e}_{1}^{\top} A_{0}^{-2} \frac{1}{T_{2}-T_{1}}\left(e^{A_{0}\left(T_{2}-t\right)}-e^{A_{0}\left(T_{1}-t\right)}\right) \mathbf{e}_{p_{0}}-\sigma_{0} \mathbf{e}_{1}^{\top} A_{0}^{-1} \mathbf{e}_{p_{0}},
$$

and

for $i=1, \ldots, n$.

$$
\bar{\Sigma}_{Y_{i}}\left(t, T_{1}, T_{2}\right)=\sigma_{i} \mathbf{e}_{1}^{\top} A_{i}^{-1} \frac{1}{T_{2}-T_{1}}\left(e^{A_{i}\left(T_{2}-t\right)}-e^{A_{i}\left(T_{1}-t\right)}\right) \mathbf{e}_{p_{i}}
$$

Proof. The result follows after a straightforward integration of $\Sigma_{X}(T-t)$ and $\Sigma_{i}(T-t)$.

We notice from the inverse of $A_{0}$ (see Lemma 4.2 in Benth and Šltytė Benth [12]) that the last term in $\bar{\Sigma}_{X}\left(t, T_{1}, T_{2}\right)$ is equal to $\sigma_{0} \mathbf{e}_{1}^{\top} A_{0}^{-1} \mathbf{e}_{p_{0}}=-\sigma_{0} / \alpha_{p_{0}}$. It may be convenient to re-parametrize the expressions for the volatilities into "time to settlement" $x=T_{1}-t$ and "length of settlement" $y=T_{2}-T_{1}$. The following corollary follows immediately from Proposition 10,

Corollary 11. For $t \geq 0, x \geq 0$ and $y \geq 0$, it holds that $\bar{\Sigma}_{X}\left(t, T_{1}, T_{2}\right)=\widetilde{\bar{\Sigma}}_{X}\left(T_{1}-t, T_{2}-T_{1}\right)$ and $\bar{\Sigma}_{Y_{i}}\left(t, T_{1}, T_{2}\right)=\widetilde{\bar{\Sigma}}_{Y_{i}}\left(T_{1}-t, T_{2}-T_{1}\right)$, where

$$
\widetilde{\bar{\Sigma}}_{X}(x, y)=\sigma_{0} y^{-1} \mathbf{e}_{1}^{\top} A_{0}^{-2} e^{A_{0} x}\left(e^{A_{0} y}-I_{p_{0}}\right) \mathbf{e}_{p_{0}}+\frac{\sigma_{0}}{\alpha_{p_{0}}},
$$

and

for $i=1, \ldots, n$.

$$
\widetilde{\bar{\Sigma}}_{Y_{i}}(x, y)=\sigma_{i} y^{-1} \mathbf{e}_{1}^{\top} A_{i}^{-1} e^{A_{i} x}\left(e^{A_{i} y}-I_{p_{i}}\right) \mathbf{e}_{p_{i}},
$$


In the procedure above, we have suggested to approximate the FFA with settlement in (32) by a geometric Brownian motion dynamics. Indeed, we claim here that the sum (i.e., the average) over geometric Brownian motions are reasonably approximated by another geometric Brownian motion. It turns out that such an approximation is relatively good, as empirically investigated in Benth [11]. Although not perfectly correct, it provides an analytically tractable dynamics for further studies.

The volatility structure of the FFA for route $i$ is defined naturally by

$$
\widetilde{\bar{\Sigma}}_{i}^{2}(x, y):=\widetilde{\bar{\Sigma}}_{X}^{2}(x, y)+2 \rho_{0, i} \widetilde{\bar{\Sigma}}_{X}(x, y) \widetilde{\bar{\Sigma}}_{Y_{i}}(x, y)+\widetilde{\bar{\Sigma}}_{Y_{i}}^{2}(x, y)
$$

Returning back to our estimated model for Supramax data, we have plotted the volatility term structure for the common factor and for route 1 in Figure 10. We assume monthly settlement period (e.g., $y=1$ month) and we plot the volatility for contracts starting settlement immediately, in one month, two months etc... The stepwise graph indicates the volatility over the settlement month. Recall that we have estimated on weekly data, so one month corresponds to 4 on our time scale. We observe that the volatility is not equal to zero

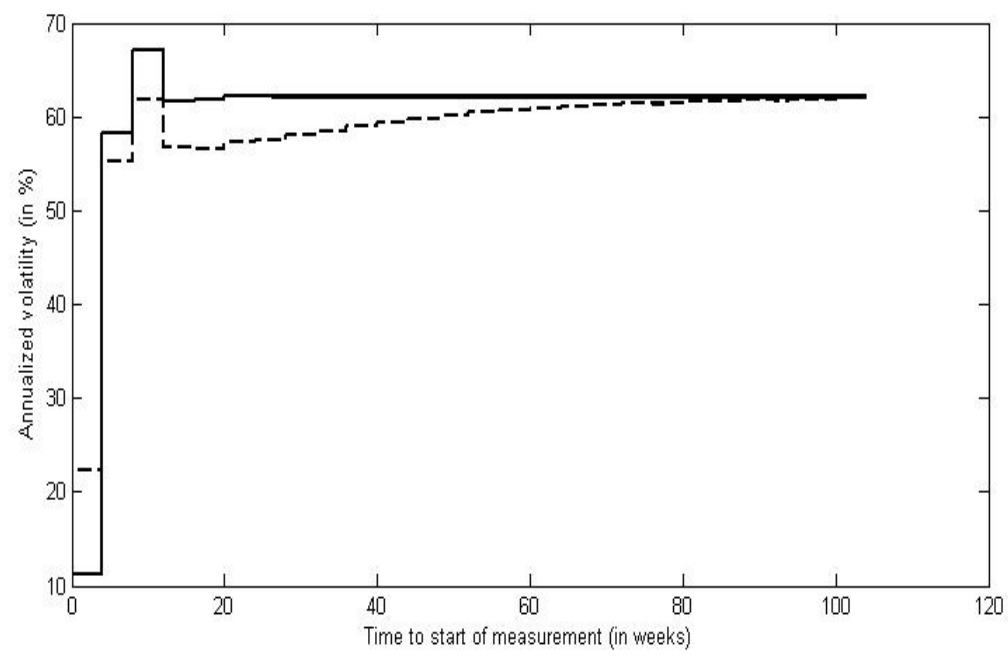

FIGURE 10. Plot of the volatility term structure for monthly contracts on route 1 (broken curve) and the common factor (solid curve) for the estimated parameters in Section 2.

for the front contract, being above $10 \%$ for the common factor and above $20 \%$ for the route. Recall the volatility term structure for instantaneous delivery in Figure 8. In Figure 11 we have plotted the correlation term structure between route 1 and the common factor. Notable is the very high positive correlation for the front month contracts, being slightly below 0.8 . Recall that for instantaneous delivery, the correlation for contracts with immediate delivery was -0.4 . This shows that the effect of having settlement periods is rather dramatic on both volatility and correlation. Due to stationarity of the factor $Y_{1}$ and cointegration, a monthly FFA on route 1 will be perfectly correlated with the common factor in the long end of the curve. Thus, for contracts on route 1 settled far out on the curve, there is approximately zero basis risk with respect to the common factor.

The FFA dynamics in (33) is a geometric Brownian motion, with an explicitly given volatility term structure in Prop. 10. Hence, we can easily price call and put options on FFAs using the Black-76 formula for time-dependent volatility, see e.g., Benth and Šaltytė Benth [12, Prop. 7.6]. Given price quotes from the market, we can calibrate the parameters of the volatility term structure from the implied volatilities. However, this procedure will not provide us with an estimate of the market price of risk vector $\gamma$ defining the pricing measure $Q$. Furthermore, although there is a trade in options on FFAs, this market is still highly illiquid. 


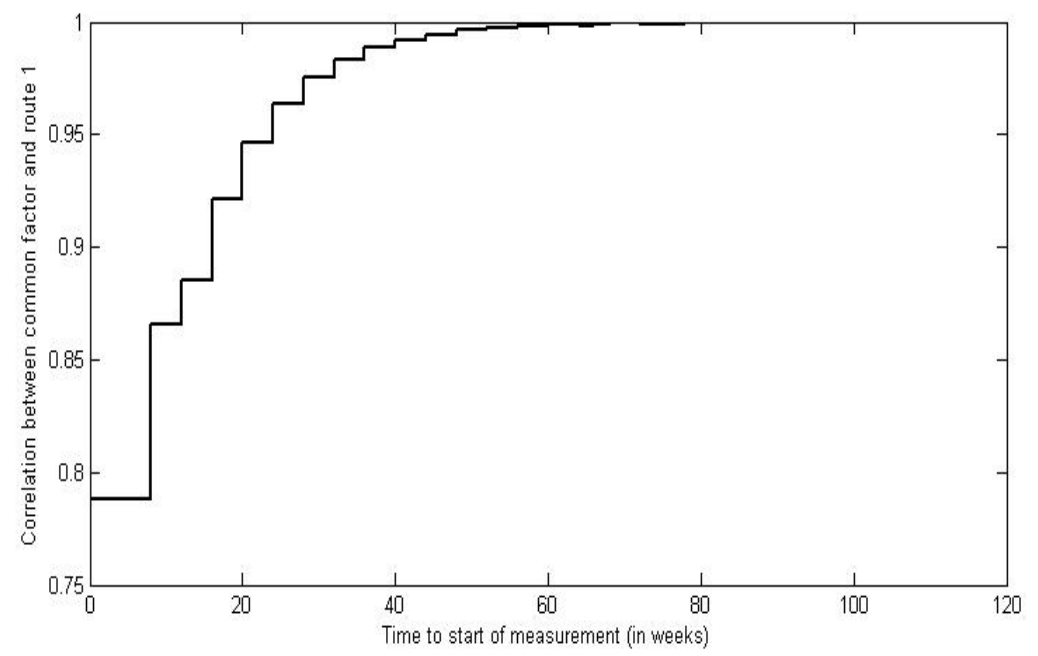

FIGURE 11. Plot of the correlation term structure between route 1 and the common factor for monthly contracts for the estimated parameters in Section 2.

\section{ApPLiCATION TO HEDGing OF REGIONAL RISK}

In this section we apply our models to a problem of hedging the risk exposure when entering positions in the physical market. In reality, FFAs are traded only on the global average tripcharter averages provided by the Baltic Exchange, while physical players typically have exposure in various route segments. This means that market agents must decide on the optimal cross-hedging strategy given their portfolio of physical contracts on the individual routes. We note that our framework can be easily expanded to any number of individual routes. We focus on two specific mean-variance optimal hedging problems, a static hedging problem and a dynamic one set in the framework of Duffie and Richardson [22].

We suppose that we have available a tradeable FFA contract written on the market factor with settlement period $\left[T_{1}, T_{2}\right]$, and assume that its risk-neutral dynamics is given by (33). Thus, with the specification of the probability $Q$ in the previous section, we find the market dynamics of the FFA to be

$$
\frac{d \bar{F}_{X}\left(t, T_{1}, T_{2}\right)}{\bar{F}_{X}\left(t, T_{1}, T_{2}\right)}=\gamma_{0} \bar{\Sigma}_{X}\left(t, T_{1}, T_{2}\right) d t+\bar{\Sigma}_{X}\left(t, T_{1}, T_{2}\right) d B_{0}(t)
$$

for $t \leq T_{1}$, and volatility $\bar{\Sigma}_{X}\left(t, T_{1}, T_{2}\right)$ given in (34). Assume that we have a risk exposure in route $i$, which is given by $k \int_{T_{1}}^{T_{2}} S_{i}(s) d s$ for $k \in \mathbb{R}$. If $k=-1$, then we have promised to pay the freight rate to a counterparty over the settlement period $\left[T_{1}, T_{2}\right]$, or equivalently, the service of shipping route $i$ in the settlement period. In practice this physical exposure could be in the form of an index-linked trip or timecharter for an individual ship, or an approximation of the cashflow from a large fleet of vessels trading on a particular route. We hedge this exposure by the FFA market forward contracts, and we consider a static hedge, that is, taking a fixed position at time $t=0$. We thus have a portfolio position with value at time $t \geq 0$,

$$
V(\pi)(t)=k \int_{T_{1}}^{T_{2}} S_{i}(s) d s+\pi\left(\bar{F}_{X}\left(t, T_{1}, T_{2}\right)-\bar{F}_{X}\left(0, T_{1}, T_{2}\right)\right) .
$$

Here, $\pi \in \mathbb{R}$ is our hedging position in the market FFA, and we suppose that we hedge up to time $T \leq T_{1}$. Hence, we do not consider any trading within the settlement period.

The aim is to maximize the value $V(\pi)(T)$ for the utility function $U(v)=v-c v^{2}$ for $c>0$ a constant measuring the risk aversion. The problem is of mean-variance type, where we seek to find $\pi^{*}$ such that

$$
\max _{\pi \in \mathbb{R}} \mathbb{E}[U(V(\pi)(T))]=\mathbb{E}\left[U\left(V\left(\pi^{*}\right)(T)\right)\right]
$$

This problem has the following solution. 
Proposition 12. The optimal static hedge $\pi^{*}$ solving (40) is

$$
\pi^{*}=\frac{\mathbb{E}\left[\bar{F}_{X}\left(T, T_{1}, T_{2}\right)\right]-\bar{F}_{X}\left(0, T_{1}, T_{2}\right)-2 c k \mathbb{E}\left[\left(\bar{F}_{X}\left(T, T_{1}, T_{2}\right)-\bar{F}_{X}\left(0, T_{1}, T_{2}\right)\right) \int_{T_{1}}^{T_{2}} S_{i}(s) d s\right]}{2 c \mathbb{E}\left[\left(\bar{F}_{X}\left(T, T_{1}, T_{2}\right)-\bar{F}_{X}\left(0, T_{1}, T_{2}\right)\right)^{2}\right]}
$$

Proof. For a function $g(\pi)=a+b \pi-\eta \pi^{2}$, where $\eta>0$ and $a, b$ two constants, we find the maximum value attained for $\pi^{*}=b / 2 \eta$. Computing $\mathbb{E}[U(V(\pi)(T))]=: g(\pi)$, we find

$$
\eta=c E\left[\left(\bar{F}_{X}\left(T, T_{1}, T_{2}\right)-\bar{F}_{X}\left(0, T_{1}, T_{2}\right)\right)^{2}\right]
$$

and

$$
b=\mathbb{E}\left[F_{X}\left(T, T_{1}, T_{2}\right)\right]-\bar{F}_{X}\left(0, T_{1}, T_{2}\right)-2 c k \mathbb{E}\left[\left(\bar{F}_{X}\left(T, T_{1}, T_{2}\right)-\bar{F}_{X}\left(0, T_{1}, T_{2}\right)\right) \int_{T_{1}}^{T_{2}} S_{i}(s) d s\right],
$$

and the result follows since $\eta>0$.

To find $\pi^{*}$, we must know $\mathbb{E}\left[\bar{F}_{X}\left(T, T_{1}, T_{2}\right)\right], \mathbb{E}\left[\bar{F}_{X}^{2}\left(T, T_{1}, T_{2}\right)\right], \mathbb{E}\left[\bar{F}_{X}\left(T, T_{1}, T_{2}\right) \int_{T_{1}}^{T_{2}} S_{i}(s) d s\right]$ and $\mathbb{E}\left[\int_{T_{1}}^{T_{2}} S_{i}(s) d s\right]$. For our model specifications, we can compute semi-analytic expressions for these expectation functionals. We list the formulas in a sequence of Lemmas.

Lemma 13. It holds that

$$
\begin{aligned}
& \mathbb{E}\left[\bar{F}_{X}\left(T, T_{1}, T_{2}\right)\right]=\bar{F}_{X}\left(0, T_{1}, T_{2}\right) \exp \left(\gamma_{0} \int_{0}^{T} \bar{\Sigma}_{X}\left(s, T_{1}, T_{2}\right) d s\right) \\
& \mathbb{E}\left[\bar{F}_{X}^{2}\left(T, T_{1}, T_{2}\right)\right]=\bar{F}_{X}^{2}\left(0, T_{1}, T_{2}\right) \exp \left(\int_{0}^{T} 2 \gamma_{0} \bar{\Sigma}_{X}\left(s, T_{1}, T_{2}\right)+\bar{\Sigma}_{X}^{2}\left(s, T_{1}, T_{2}\right) d s\right)
\end{aligned}
$$

Proof. Since

$$
\begin{aligned}
& \bar{F}_{X}\left(T, T_{1}, T_{2}\right)=\bar{F}_{X}\left(0, T_{1}, T_{2}\right) \exp \left(\int_{0}^{T} \gamma_{0} \bar{\Sigma}_{X}\left(s, T_{1}, T_{2}\right)-\frac{1}{2} \bar{\Sigma}_{X}^{2}\left(s, T_{1}, T_{2}\right) d s\right) \\
& \times \exp \left(\int_{0}^{T} \bar{\Sigma}_{X}\left(s, T_{1}, T_{2}\right) d B_{0}(s)\right)
\end{aligned}
$$

the expressions follow from standard formulas for exponential moments of Gaussian random variables.

Lemma 14. It holds that

$$
\mathbb{E}\left[\int_{T_{1}}^{T_{2}} S_{i}(s) d s\right]=\int_{T_{1}}^{T_{2}} \exp \left(m(s)+\frac{1}{2} w(s)\right) d s
$$

where

$$
m(s):=\mathbf{e}_{1, i}^{\top} e^{A_{i} s} \mathbf{Z}_{i}(0)+\mathbf{e}_{1,0}^{\top} A_{0}^{-1}\left(e^{A_{0} s}-I\right) \mathbf{Z}_{0}(0)
$$

and

$$
\begin{aligned}
w(s):= & \sigma_{i}^{2} \int_{0}^{s} \mathbf{e}_{p_{i}}^{\top} e^{A_{i}^{\top} v} \mathbf{e}_{1, i} \mathbf{e}_{1, i}^{\top} e^{A_{i} v} \mathbf{e}_{p_{i}} d v+2 \sigma_{0} \sigma_{i} \rho_{0, i} \int_{0}^{s} \mathbf{e}_{p_{i}}^{\top} e^{A_{i} v} \mathbf{e}_{1, i} \mathbf{e}_{1,0}^{\top} A_{0}^{-1}\left(e^{A_{0} v}-I\right) \mathbf{e}_{p_{0}} d v \\
& +\sigma_{0}^{2} \int_{0}^{s} \mathbf{e}_{p_{0}}^{\top}\left(e^{A_{0}^{\top} v}-I\right) A_{0}^{-\top} \mathbf{e}_{1,0} \mathbf{e}_{1,0}^{\top} A_{0}^{-1}\left(e^{A_{0} v}-I\right) \mathbf{e}_{p_{0}} d v .
\end{aligned}
$$

Here we have used the notation $\mathbf{e}_{1, i}$ and $\mathbf{e}_{1,0}$ to distinguish the first unit vectors in $\mathbb{R}^{p_{i}}$ and $\mathbb{R}^{p_{0}}$, resp.

Proof. From the definition of $S_{i}(s)$, we find

$$
\mathbb{E}\left[\int_{T_{1}}^{T_{2}} S_{i}(s) d s\right]=e^{\theta_{i}} \int_{T_{1}}^{T_{2}} \mathbb{E}\left[\exp \left(\int_{0}^{s} Y_{0}(u) d u+Y_{i}(s)\right)\right] d s .
$$

We recall that

$$
Y_{i}(s)=\mathbf{e}_{1}^{\top} e^{A_{i} s} \mathbf{Z}_{i}(0)+\sigma_{i} \int_{0}^{s} \mathbf{e}_{1}^{\top} e^{A_{i}(s-v)} \mathbf{e}_{p_{i}} d B_{i}(v) .
$$


Note that $\mathbf{e}_{1}$ is the first unit vector in $\mathbb{R}^{p_{i}}$. Furthermore, using the analogous expression for $Y_{0}(u)$, we derive using the stochastic Fubini theorem,

$$
\begin{aligned}
\int_{0}^{s} Y_{0}(u) d u & =\mathbf{e}_{1}^{\top} \int_{0}^{s} e^{A_{0} u} d u \mathbf{Z}_{0}(0)+\sigma_{0} \int_{0}^{s} \int_{0}^{u} \mathbf{e}_{1}^{\top} e^{A_{0}(u-v)} \mathbf{e}_{p_{0}} d B_{0}(v) d u \\
& =\mathbf{e}_{1}^{\top} A_{0}^{-1}\left(e^{A_{0} s}-I\right) \mathbf{Z}_{0}(0)+\sigma_{0} \int_{0}^{s} \mathbf{e}_{1}^{\top} A_{0}^{-1}\left(e^{A_{0}(s-v)}-I\right) \mathbf{e}_{p_{0}} d B_{0}(v) .
\end{aligned}
$$

Here, $\mathbf{e}_{1}$ is the first unit vector in $\mathbb{R}^{p_{0}}$. Hence, $\int_{0}^{s} Y_{0}(u) d u+Y_{i}(s)$ is a normally distributed random variable with mean $m(s)$ and variance $w(s)$. The result follows.

Lemma 15. It holds,

$$
\mathbb{E}\left[\bar{F}_{X}\left(T, T_{1}, T_{2}\right) \int_{T_{1}}^{T_{2}} S_{i}(s) d s\right]=\bar{F}_{X}\left(0, T_{1}, T_{2}\right) e^{\theta_{i}} \int_{T_{1}}^{T_{2}} \exp \left(\widetilde{m}_{T}(s)+\frac{1}{2} \widetilde{w}_{T}(s)\right) d s
$$

where

$$
\widetilde{m}_{T}(s):=\int_{0}^{T} \gamma_{0} \bar{\Sigma}_{X}\left(v, T_{1}, T_{2}\right)-\frac{1}{2} \bar{\Sigma}_{X}^{2}\left(v, T_{1}, T_{2}\right) d v+\mathbf{e}_{1, i}^{\top} e^{A_{i} s} \mathbf{Z}_{i}(0)+\mathbf{e}_{1,0}^{\top} A_{0}^{-1}\left(e^{A_{0} s}-I\right) \mathbf{Z}_{0}(0),
$$

and

$$
\begin{aligned}
\widetilde{w}_{T}(s):= & \sigma_{i}^{2} \int_{0}^{s} \mathbf{e}_{p_{i}}^{\top} e^{A_{i}^{\top} v} \mathbf{e}_{1, i} \mathbf{e}_{1, i}^{\top} e^{A_{i} v} \mathbf{e}_{p_{i}} d v \\
& +2 \sigma_{i} \rho_{0, i} \int_{0}^{s} \mathbf{e}_{p_{i}}^{\top} e^{A_{i}^{\top} v} \mathbf{e}_{1, i}\left(\mathbf{1}_{[0, T]}(v) \bar{\Sigma}_{X}\left(v, T_{1}, T_{2}\right)+\sigma_{0} \mathbf{e}_{1,0}^{\top} A_{0}^{-1}\left(e^{A_{0} v}-I\right) \mathbf{e}_{p_{0}}\right) d v \\
& +\int_{0}^{s}\left(\mathbf{1}_{[0, T]}(v) \bar{\Sigma}_{X}\left(v, T_{1}, T_{2}\right)+\sigma_{0} \mathbf{e}_{1,0}^{\top} A_{0}^{-1}\left(e^{A_{0} v}-I\right) \mathbf{e}_{p_{0}}\right)^{2} d v .
\end{aligned}
$$

Proof. Using the explicit dynamics of $\bar{F}_{X}\left(t, T_{1}, T_{2}\right)$ and $S_{i}(s)$ we find,

$$
\begin{aligned}
& \mathbb{E}\left[\bar{F}_{X}\left(T, T_{1}, T_{2}\right) \int_{T_{1}}^{T_{2}} S_{i}(s) d s\right] \\
& =\int_{T_{1}}^{T_{2}} \mathbb{E}\left[\bar{F}_{X}\left(T, T_{1}, T_{2}\right) S_{i}(s)\right] d s \\
& =\bar{F}_{X}\left(0, T_{1}, T_{2}\right) e^{\theta_{i}} \int_{T_{1}}^{T_{2}} \exp \left(\int_{0}^{T} \gamma_{0} \bar{\Sigma}_{X}\left(v, T_{1}, T_{2}\right)-\frac{1}{2} \bar{\Sigma}_{X}\left(v, T_{1}, T_{2}\right) d v\right) \\
& \quad \times \mathbb{E}\left[\exp \left(\int_{0}^{T} \bar{\Sigma}_{X}\left(v, T_{1}, T_{2}\right) d B_{0}(v)+\int_{0}^{s} Y_{0}(u) d u+Y_{i}(s)\right)\right] d s
\end{aligned}
$$

Following the same analysis as in the proof of Lemma 14 yields the result.

With these three lemmas at hand, we can find an expression for $\pi^{*}$ in Proposition 12 in terms of integrals of the various components in the dynamics of route $i$ and the FFA forward price. In a concrete application, we must perform numerical integration to find the explicit optimal static hedge.

If we want to compute the optimal expected value function, we need in addition $\mathbb{E}\left[\left(\int_{T_{1}}^{T_{2}} S_{i}(s) d s\right)^{2}\right]$, which is stated in the next Lemma:

Lemma 16. It holds,

$$
\mathbb{E}\left[\left(\int_{T_{1}}^{T_{2}} S_{i}(s) d s\right)^{2}\right]=2 e^{2 \theta_{i}} \int_{T_{1}}^{T_{2}} \int_{T_{1}}^{t} \exp \left(\bar{m}(t, s)+\frac{1}{2} \bar{w}(t, s)\right) d s d t
$$

where, for $s \leq t$,

$$
\bar{m}(t, s):=\mathbf{e}_{1, i}^{\top}\left(e^{A_{i} s}+e^{A_{i} t}\right) \mathbf{Z}_{i}(0)+\mathbf{e}_{1,0}^{\top} A_{0}^{-1}\left(e^{A_{0} s}+e^{A_{0} t}-2 I\right) \mathbf{Z}_{0}(0),
$$


and

$$
\begin{aligned}
\bar{w}(t, s):= & \sigma_{i}^{2} \int_{0}^{t}\left(\mathbf{e}_{1, i}^{\top}\left(e^{A_{i}(s-v)} \mathbf{1}_{[0, s]}(v)+e^{A_{i}(t-v)}\right) \mathbf{e}_{p_{i}}\right)^{2} d v \\
& +2 \sigma_{0} \sigma_{0} \rho_{0, i} \int_{0}^{t}\left(\mathbf{e}_{1, i}^{\top}\left(e^{A_{i}(s-v)} \mathbf{1}_{[0, s]}(v)+e^{A_{i}(t-v)}\right) \mathbf{e}_{p_{i}}\right) \\
& \times\left(\mathbf{e}_{1,0}^{\top} A_{0}^{-1}\left(\left(e^{A_{0}(s-v)}-I\right) \mathbf{1}_{[0, s]}(v)+\left(e^{A_{0}(t-v)}-I\right)\right) \mathbf{e}_{p_{0}}\right) d v \\
& +\sigma_{0}^{2} \int_{0}^{t}\left(\mathbf{e}_{1,0}^{\top} A_{0}^{-1}\left(\left(e^{A_{0}(s-v)}-I\right) \mathbf{1}_{[0, s]}(v)+\left(e^{A_{0}(t-v)}-I\right)\right) \mathbf{e}_{p_{0}}\right)^{2} d v .
\end{aligned}
$$

Proof. Since

$$
\left(\int_{T_{1}}^{T_{2}} S_{i}(s) d s\right)^{2}=2 \int_{T_{1}}^{T_{2}} \int_{T_{1}}^{t} S_{i}(s) S_{i}(t) d s d t
$$

it holds

$$
\begin{aligned}
& \mathbb{E}\left[\left(\int_{T_{1}}^{T_{2}} S_{i}(s) d s\right)^{2}\right] \\
& \quad=2 \int_{T_{1}}^{T_{2}} \int_{T_{1}}^{t} \mathbb{E}\left[S_{i}(s) S_{i}(t)\right] d s d t \\
& \quad=2 e^{2 \theta_{i}} \int_{T_{1}}^{T_{2}} \int_{T_{1}}^{t} \mathbb{E}\left[\exp \left(\int_{0}^{s} Y_{0}(u) d u+Y_{i}(s)+\int_{0}^{t} Y_{0}(u) d u+Y_{i}(t)\right)\right] d s d t .
\end{aligned}
$$

Using the expressions for $\int_{0}^{s} Y_{0}(u) d u$ and $Y_{i}(s)$ derived in the proof of Lemma 14, we can conclude the result by appealing to the known expressions for the exponential moment of normal random variables.

We end this first mean-variance hedging application by remarking that one may extend the above analysis to include hedging within the settlement period by modifying the dynamics of the FFAs. We refer to Benth and Dethering [14] for an analysis of dynamic hedging when one cannot hedge within a settlement period.

We now move our attention to a slightly different mean-variance problem, which can be formulated in the context of Duffie and Richardson [22]. Suppose that a trader has an exposure in rute $i$ given by $k \bar{F}_{i}\left(T, \tau_{1}, \tau_{2}\right)$ for $T \leq \tau_{1}$ and $k$ some real number (positive or negative, indicating long or short position in the FFA). This FFA is not traded, but we aim at hedging the risk exposure by dynamically trading in the market FFA. I.e., we consider a situation where we aim at matching a fixed FFA position in route $i$ (which is not tradeable) with dynamically trading in the market FFA. In some sense, we can view this problem as trying to mimic a FFA in route $i$ using the market FFA.

Recall the dynamics of $\bar{F}_{i}\left(t, \tau_{1}, \tau_{2}\right)$ from (35) to be (with respect to the market probability),

$$
\begin{aligned}
\frac{d \bar{F}_{i}\left(t, \tau_{1}, \tau_{2}\right)}{\bar{F}_{i}\left(t, \tau_{1}, \tau_{2}\right)}=( & \left.\gamma_{0} \bar{\Sigma}_{X}\left(t, \tau_{1}, \tau_{2}\right)+\gamma_{i} \bar{\Sigma}_{Y_{i}}\left(t, \tau_{1}, \tau_{2}\right)\right) d t \\
& +\bar{\Sigma}_{X}\left(t, \tau_{1}, \tau_{2}\right) d B_{0}(t)+\bar{\Sigma}_{Y_{i}}\left(t, \tau_{1}, \tau_{2}\right) d B_{i}(t),
\end{aligned}
$$

where $\bar{\Sigma}_{Y_{i}}\left(t, \tau_{1}, \tau_{2}\right)$ is defined in (36). We suppose here that the start of the settlement period of the market FFA is after the settlement of the regional FFA, that is, $\tau_{1} \leq T_{1} \leq \tau_{2}$. This is for convenience since we have not studied the FFA price dynamics within the settlement period, and if we would have $T \geq T_{1}$, say, then we would need to use the market FFA in the hedging procedure also within its settlement. However, it is not too difficult to dispense with this restriction by, for example, choosing another market FFA with later settlement, or to consider a roll-over strategy (which we do not consider here).

We suppose that the trader dynamically hedge in the market FFA using an $\mathcal{F}_{t}$-adapted strategy $\pi(t)$ which is integrable with respect to $d \bar{F}_{X}\left(t, T_{1}, T_{2}\right)$. The aim for the trader is to maximize the wealth at time $T$, defined by $V(\pi)(T)$ with

$$
V(\pi)(t):=k \bar{F}_{i}\left(T, \tau_{1}, \tau_{2}\right)+\int_{0}^{t} \pi(s) d \bar{F}_{X}\left(s, T_{1}, T_{2}\right) .
$$


The utility function is again $U(v)=v-c v^{2}$ for some risk-aversion coefficient $c>0$, and the problem of the trader is to find an optimal $\pi^{*}$ maximizing $\mathbb{E}[U(V(\pi)(T))]$. The solution to this problem is given in Duffie and Richardson [22]. After some reformulation and identification, we find from their paper that the optimal $\pi^{*}$ is given as follows:

Define the function $\alpha(t, T)$ for $t \leq T$ by

$$
\begin{aligned}
\alpha(t, T)=k & \frac{\gamma_{0}+\bar{\Sigma}_{X}\left(t, \tau_{1}, \tau_{2}\right)+\rho_{0, i} \bar{\Sigma}_{Y_{i}}\left(t, \tau_{1}, \tau_{2}\right)}{\bar{\Sigma}_{X}\left(t, T_{1}, T_{2}\right)} \\
& \times \exp \left(-\int_{t}^{T}\left(\gamma_{i}+\gamma_{0} \rho_{0, i}\right) \bar{\Sigma}_{Y_{i}}\left(s, \tau_{1}, \tau_{2}\right)+2 \gamma_{0} \bar{\Sigma}_{X}\left(s, \tau_{1}, \tau_{2}\right) d s\right) .
\end{aligned}
$$

Then we have $\pi^{*}$ given by the equation

$$
\begin{array}{r}
\pi^{*}(t) \bar{F}_{X}\left(t, T_{1}, T_{2}\right)+\frac{\gamma_{0}}{\bar{\Sigma}_{X}\left(t, T_{1}, T_{2}\right)} \int_{0}^{t} \pi^{*}(s) d \bar{F}_{X}\left(s, T_{1}, T_{2}\right) \\
=\frac{\gamma_{0}}{2 c \bar{\Sigma}_{X}\left(t, T_{1}, T_{2}\right)}-\alpha(t, T) \bar{F}_{i}\left(t, \tau_{1}, \tau_{2}\right)
\end{array}
$$

The optimal strategy $\pi^{*}$ is given through an integral equation, which can be solved using numerical simulation in a discretized form. Choosing a homogeneous time stepping $t_{n}=n \Delta, n=0,1, \ldots, N$ with $N \in \mathbb{N}$ and $\Delta:=T / N$ (where in practice $T>>N$, of course), we find the recursive scheme based on a Riemann approximation of the integral

$$
\begin{aligned}
& \pi^{*}\left(t_{n}\right) \bar{F}_{X}\left(t_{n}, T_{1}, T_{2}\right)+ \frac{\gamma_{0}}{\bar{\Sigma}_{X}\left(t_{n}, T_{1}, T_{2}\right)} \sum_{i=0}^{n-1} \pi^{*}\left(t_{i}\right) \Delta \bar{F}_{X}\left(t_{i}, T_{1}, T_{2}\right) \\
&=\frac{\gamma_{0}}{2 c \bar{\Sigma}_{X}\left(t_{n}, T_{1}, T_{2}\right)}-\alpha\left(t_{n}, T\right) \bar{F}_{i}\left(t_{n}, \tau_{1}, \tau_{2}\right) .
\end{aligned}
$$

Here, $\Delta \bar{F}_{X}\left(t_{i}, T_{1}, T_{2}\right):=\bar{F}_{X}\left(t_{i+1}, T_{1}, T_{2}\right)-\bar{F}_{X}\left(t_{i}, T_{1}, T_{2}\right)$. Given $\pi^{*}(0)$, we can iterate the above recursion to compute $\pi^{*}\left(t_{n}\right)$ for all $n=1, \ldots, N$. By (44), we find

$$
\pi^{*}(0)=\frac{\gamma_{0}}{2 c \bar{\Sigma}_{X}\left(0, T_{1}, T_{2}\right) \bar{F}_{X}\left(0, T_{1}, T_{2}\right)}-\alpha(0, T) \frac{\bar{F}_{i}\left(0, \tau_{1}, \tau_{2}\right)}{\bar{F}_{X}\left(0, T_{1}, T_{2}\right)} .
$$

To run this numerical scheme, we must have available the prices $\bar{F}_{i}\left(t_{i}, \tau_{1}, \tau_{2}\right)$. As the market does not trade in the regional FFAs, we can instead apply the derived regional FFA dynamics in the previous Section as benchmarking using the observed spot freight rates for the regional route.

\section{AN OUTLOOK TO NON-GAUSSIAN MODELS}

There is evidence for jumps in freight prices (see e.g. Nomikos et al. [36] and Kyriakou et al. [33]), and more general dynamics than the Brownian-driven models analysed in this paper may be desirable. In this Section we briefly discuss extensions of our models, both in view of some exisiting literature on non-Gaussian dynamics for financial time series and in the context of our specific Supramax data.

An alternative to the classical (multivariate) geometric Brownian motion dynamics is a multidimensional exponential Lévy process (see e.g. Ballotta and Bonfiglioli [5]). A Lévy process allows for much more flexibility to include jumps in the price dynamics and to model heavy tails and skewness in returns. On the other hand, Lévy processes preserve analytical tractability when it comes to pricing of derivatives.

In our context, we have argued for cointegration of Supramax regional prices, non-stationarity in the common factor as well as autoregressive effects (recall the empirical analysis in Section 3). We consider here substituting the Brownian driver in the $\operatorname{CAR}(p)$-dynamics with a Lévy process. To this end, let $\mathbf{L}=$ $\left(L_{0}, L_{1}, \ldots, L_{n}\right)^{\top}$ be a $n+1$-dimensional Lévy process where we assume that the moment generating function exists for all $\mathbf{x} \in \Theta, \Theta$ being an open subset of $\mathbb{R}^{n+1}$ including the origin (see Applebaum [3]). We introduce the log-moment generating function

$$
\phi(\mathbf{x})=\ln \mathbb{E}\left[\exp \left(\mathbf{x}^{\top} \mathbf{L}(1)\right)\right], \mathbf{x} \in \mathbf{\Theta} .
$$


We note in passing that the Lévy process has finite second moment. From the Levy-Kintchine representation, we find

$$
\phi(\mathbf{x})=\mathbf{x}^{\top} \mathbf{a} t+\frac{1}{2} \mathbf{x}^{\top} C \mathbf{x}+\int_{\mathbb{R}^{n+1} \backslash\{0\}}\left(e^{\mathbf{x}^{\top} \mathbf{v}}-1-\mathbf{x}^{\top} \mathbf{v}\right) \ell(d \mathbf{v})
$$

where $\mathbf{a} \in \mathbb{R}^{n+1}$ is the drift, $C \in \mathbb{R}^{(n+1) \times(n+1)}$ the covariance matrix of the Brownian motion part and $\ell$ is the Lévy measure.

Observe that $\phi_{i}(x):=\phi\left(x \mathbf{e}_{i}\right), x \in \mathbb{R}$ defines the log-moment generating function of the Lévy process $L_{i}, i=0,1, \ldots, n$, as long as $x \mathbf{e}_{i} \in \boldsymbol{\Theta}$. We define the vector-valued CAR(p)-process $\mathbf{Y}=$ $\left(Y_{0}, Y_{1}, \ldots, Y_{n}\right)$ by $Y_{i}(t)=\mathbf{e}_{1}^{\top} \mathbf{Z}_{i}(t)$, where

$$
d \mathbf{Z}_{i}(t)=A_{i} \mathbf{Z}_{i}(t) d t+\mathbf{e}_{p_{i}} d L_{i}(t)
$$

for $i=0, \ldots, n$. The matrices $A_{i}, i=0, \ldots, n$ are defined in (2) with the same assumptions as stated in Section 2. We recall the model for the regional freight rate dynamics $S_{i}(t)$ in (10) and (11).

In Figure 12 we show the empirical density of the residuals $\epsilon_{x, t}$ and $\epsilon_{i, t}, i=1,2,3,4$ in the ARdynamics of the market factor and the routes, see (16) and (17), along with the fitted normal distribution (broken line). The density plots make use of a logarithmic frequency axis to emphasise possible heavy tails on the data. As we can see, there are some deviations from Gaussianity visible in the data. In particular, all the routes and the market factor have slightly heavier tails than the normal. Also, the center of the empirical distributions have more mass than assigned by the normal distribution.
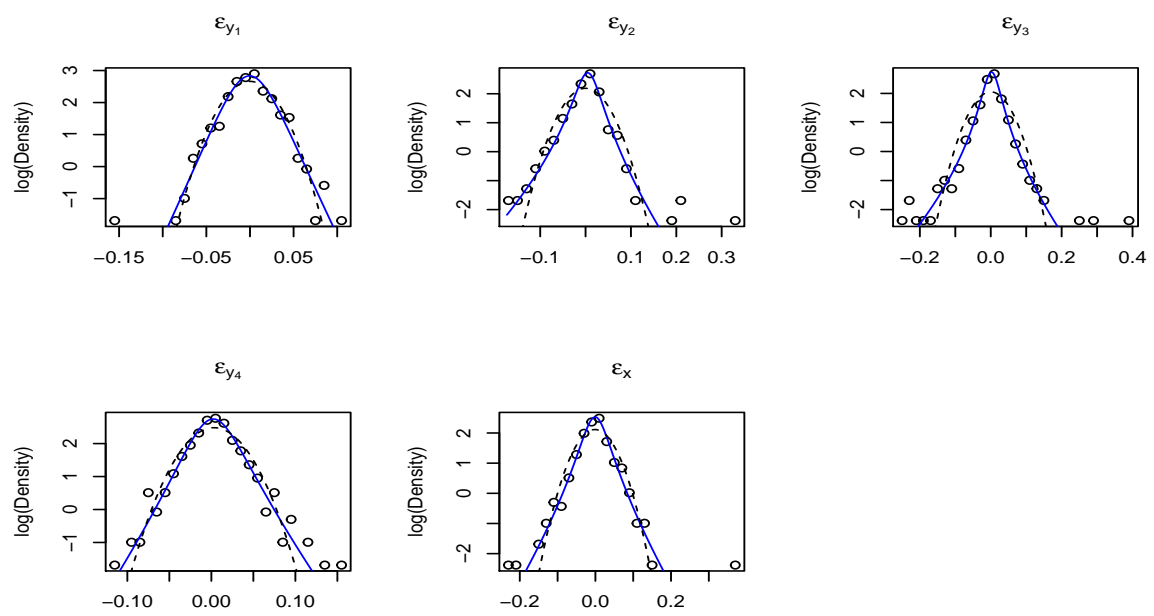

FIGURE 12. Empirical distribution of residuals with fitted normal (broken line) and NIG distribution (complete line), on a logarithmic frequency axis.

Also plotted in Figure 12 are the fitted normal inverse Gaussian (NIG) distributions estimated using maximum likelihood (complete line). The four parameter family of NIG distributions were introduced by Barndorff-Nielsen [7] as a member of the family of generalized hyperbolic distributions, which has been succesfully applied to model financial logreturns (see e.g. Barndorff-Nielsen [8] and Eberlein and Keller [23]) due to its flexibility to explain skewness and heavy tails. As we can see from the figure, the empirical distribution is better explained by the NIG than the Gaussian distribution, although the improvement is not very prominent.

The log-moment generating function of an NIG-distributed random variable takes the form (see BarndorffNielsen [8])

$$
\phi_{\mathrm{NIG}}(x)=\mu x+\delta\left(\sqrt{\alpha^{2}-\beta^{2}}-\sqrt{\alpha^{2}-(\beta+x)^{2}}\right)
$$


where $\alpha>|\beta|$ and $x \in \mathbb{R}$ such that $\alpha>|\beta+x|$. The parameters are named $\mu \in \mathbb{R}$ location, $\beta \in \mathbb{R}$ skewness, ${ }^{3} \alpha>0$ tail heaviness and $\delta>0$ scale. A multivariate $(n+1$-dimensional) NIG distribution is introduced in Rydberg [41] (see also Øigård and Hansen [37]), which has the log-moment generating function

$$
\phi_{\mathrm{MNIG}}(\mathbf{x})=\mathbf{x}^{\top} \boldsymbol{\mu}+\delta\left(\sqrt{\alpha^{2}-\boldsymbol{\beta}^{\top} \Gamma \boldsymbol{\beta}}-\sqrt{\alpha^{2}-(\boldsymbol{\beta}+\mathbf{x})^{\top} \Gamma(\boldsymbol{\beta}+\mathbf{x})}\right)
$$

Here, $\alpha, \delta>0, \boldsymbol{\mu}, \boldsymbol{\beta} \in \mathbb{R}^{n+1}$ and $\alpha^{2}>\boldsymbol{\beta}^{\top} \Gamma \boldsymbol{\beta}$. The covariance of the $n+1$-dimensional NIG distribution is described by the matrix $\Gamma \in \mathbb{R}^{(n+1) \times(n+1)}$, and the log-moment generating function $\phi_{\text {MNIG }}$ is defined for all $\mathbf{x} \in \mathbb{R}^{n+1}$ such that $\alpha^{2}>(\boldsymbol{\beta}+\mathbf{x})^{\top} \Gamma(\boldsymbol{\beta}+\mathbf{x})$.

As an application of the Lévy process model for Supramax price dynamics, let us consider pricing of a futures. Introduce a pricing measure $Q \sim P$ by the Esscher transform (see e.g. Benth, Šaltyte Benth and Koekebakker [13, Sect. 4.1]), where for a market price of risk $\gamma \in \boldsymbol{\Theta}, \mathbf{L}$ is a Lévy process in $\mathbb{R}^{n+1}$ with respect to $Q$ with log-moment generating function

$$
\phi^{\gamma}(\mathbf{x}):=\phi(\mathbf{x}+\gamma)-\phi(\gamma) .
$$

We find the following result for the futures price $F_{X}(t, T), t \leq T$ in the current context:

Proposition 17. Suppose the common factor $X$ is defined as in (11) with $\mathbf{Z}_{0}$ given as in (46). Assume that $\gamma \in \boldsymbol{\Theta}$ and $\boldsymbol{\gamma}+g(u) \mathbf{e}_{1} \in \boldsymbol{\Theta}$ for all $0 \leq u \leq T$, where

$$
g(u)=\mathbf{e}_{1}^{\top} A_{0}^{-1}\left(e^{A_{0}(T-t)}-I_{p_{0}}\right) \mathbf{e}_{p_{0}}, u \geq 0 .
$$

Then, for $0 \leq t \leq T$ it holds

$$
F_{X}(t, T)=S(t) \exp \left(\widetilde{h}\left(\mathbf{Z}_{0}(t), T-t\right)\right)
$$

where

$$
\widetilde{h}(\mathbf{z}, s)=\mathbf{e}_{1}^{\top} A_{0}^{-1}\left(e^{A_{0}(T-t)}-I_{p_{0}}\right) \mathbf{z}+\int_{0}^{s} \phi^{\gamma}\left(g(u) \mathbf{e}_{1}\right) d u
$$

for $s \geq 0, \mathbf{z} \in \mathbb{R}^{p_{0}}$, and $\phi^{\gamma}$ defined in (49).

Proof. First, observe that

$$
S(T)=\exp \left(\int_{0}^{T} Y_{0}(s) d s\right)=S(t) \exp \left(\int_{t}^{T} Y_{0}(s) d s\right) .
$$

But, since for $s \geq t$,

$$
\mathbf{Z}_{0}(s)=e^{A_{0}(s-t)} \mathbf{Z}_{0}(t)+\int_{t}^{s} e^{A_{0}(u-t)} \mathbf{e}_{p_{0}} d L_{0}(u)
$$

we find

$$
\begin{aligned}
\int_{t}^{T} Y_{0}(s) d s & =\mathbf{e}_{1}^{\top} \int_{t}^{T} \mathbf{Z}_{0}(s) d s \\
& =\mathbf{e}_{1}^{\top} A_{0}^{-1}\left(e^{A_{0}(T-t)}-I_{p_{0}}\right) \mathbf{Z}_{0}(t)+\int_{t}^{T} \mathbf{e}_{1}^{\top} A_{0}^{-1}\left(e^{A_{0}(T-u)}-I_{p_{0}}\right) \mathbf{e}_{p_{0}} d L_{0}(u)
\end{aligned}
$$

after using the stochastic Fubini Theorem. From $\mathcal{F}_{t}$-measurability of $S(t)$ and independent increment property of Lévy processes, it follows that

$$
\begin{aligned}
& F_{X}(t, T)=S(t) \exp \left(\mathbf{e}_{1}^{\top} A_{0}^{-1}\left(e^{A_{0}(T-t)}-I_{p_{0}}\right) \mathbf{Z}_{0}(t)\right) \\
& \times \mathbb{E}_{Q}\left[\exp \left(\int_{t}^{T} \mathbf{e}_{1}^{\top} A_{0}^{-1}\left(e^{A_{0}(T-u)}-I_{p_{0}}\right) \mathbf{e}_{p_{0}} d L_{0}(u)\right)\right] .
\end{aligned}
$$

\footnotetext{
${ }^{3}$ There is a slight notational clash here with the autoregression coefficients of the time series model.
} 
Calculating the expectation under $Q$ yields,

$$
\mathbb{E}_{Q}\left[\exp \left(\int_{t}^{T} \mathbf{e}_{1}^{\top} A_{0}^{-1}\left(e^{A_{0}(T-u)}-I_{p_{0}}\right) \mathbf{e}_{p_{0}} d L_{0}(u)\right)\right]=\exp \left(\int_{t}^{T} \phi^{\gamma}\left(g(T-u) \mathbf{e}_{1}\right) d u\right),
$$

and the result follows.

We remark that we can use similar arguments to derive futures price dynamics for the regional routes.

Stochastic volatility is another feature that is frequently analysed and modelled in financial time series. In our autoregressive time series for the market factor and the four routes, we have assumed independent residuals. Looking at the autocorrelation plot in Figure 13 (top), the residuals are uncorrelated (dotted lines indicate $95 \%$ significance level), whereas the squared residuals show some degree of correlation at lags up to 15-20. Although the evidence is not very strong, there are indications of dependency in the residuals. In Benth [10], a stochastic volatility model in a mean-reverting dynamics has been proposed and
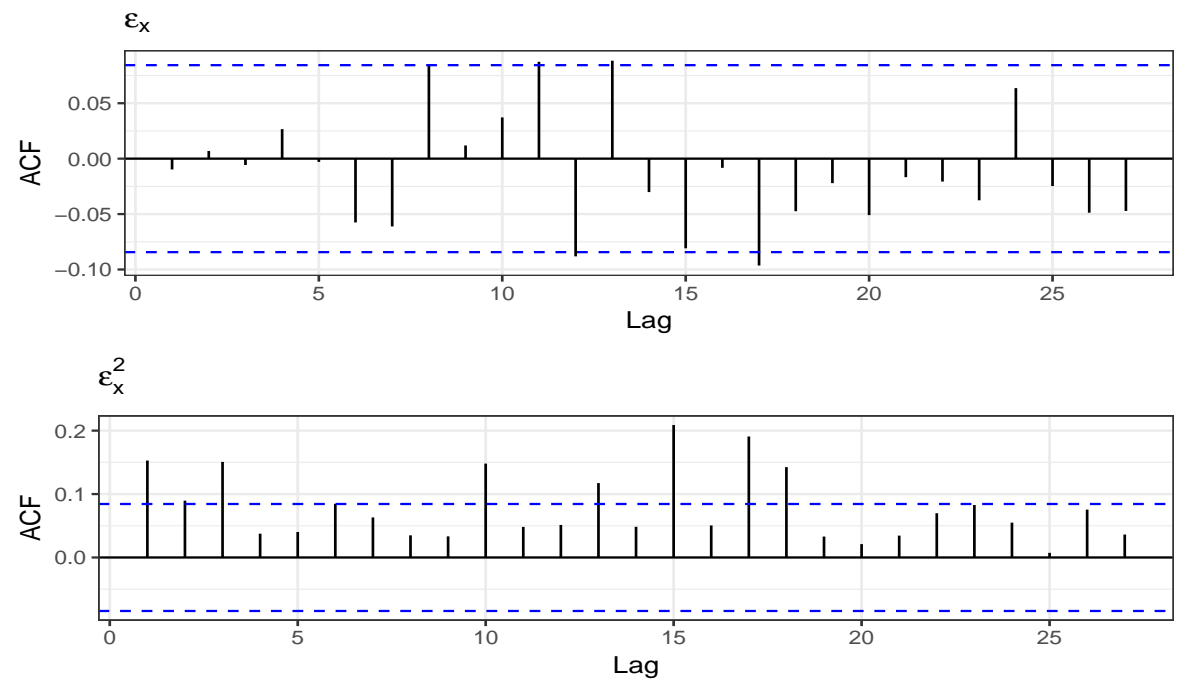

FIGURE 13. Autocorrelation function for the residuals (top) and squared residuals (bottom) for the market factor.

analysed. The stochastic volatility dynamics were assumed to follow the Barndorff-Nielsen and Shepard model (see Barndorff-Nielsen and Shephard [9]). The mean-reversion process would be a continuous-time autoregressive process or order 1 in our context. This stochastic volatility dynamics can be tailormade to explain correlation effects in the autocorrelation function along with NIG distributed residuals.

\section{CONCLusions}

In this paper we propose the decomposition of regional freight rates into a non-stationary market factor, which is observable and has a natural interpretation as the global market average, and (additive) correlated regional factors.

Our discrete-time model for the joint dynamics of regional spot freight rates is parsimonious, easy to estimate within a standard ARIMA framework, and is able to capture the main characteristics discussed in the literature. This includes: i) a common stochastic, non-stationary, trend reflecting changes in the global supply/demand balance, ii) mean-reverting deviations from the market average in regional freight rates reflecting the mobility of tonnage, iii) the long-run co-integration of regional routes and iv) the Atlantic freight rate premium (see, for instance, Berg-Andreassen [16] and Adland et al. [1]).

Based on our empirical results we can also make some interesting observations about the joint dynamics of regional freight rates. Mainly this relates to the statistical significance of the constants in our estimated regional factor models. Among our four main routes, some are consistently above or below the market rate throughout our sample. On the face of it, this is not consistent with a spatially efficient market with 
mobile assets controlled by profit-maximizing operators - in such a system we would expect the longrun average regional factor to be zero. Upon closer inspection, some but not all of this can be explained away. Specifically, we notice that in the case of the cross-basin (Fronthaul/Backhaul) routes, the constants are similar in magnitude but of opposite signs. In order to avoid building up a surplus of ships in the Pacific, we note that the flow of ships must be bi-directional and of the same magnitude. Put differently, in order to take advantage of the higher-paying Fronthaul route, the average owner must at some stage return the vessel by way of the lower-paying Backhaul route and the two effects cancel out according to our estimates. Accordingly, statistically significant regional factor constants for Routes S1 and S3 are not inconsistent with market efficiency.

The trans-Atlantic premium (S4) is well known in the literature (Adland et al. [1]) but is harder to explain. It is possible that, in reality, the availability of cargoes is lower in the Atlantic and therefore the apparent premium in rates is offset by more idle time for the vessels viz-a-viz operating the ships in the Pacific. Since our model does not account for idle time between contracts, we cannot exclude the possibility that real vessel earnings in the trans-Atlantic and trans-Pacific are in fact the same.

Future research along these lines should include further empirical applications of the hedging procedures described herein. While our model has been derived with the spot freight market in mind, it is applicable to any cross-hedging problem where agents have physical exposure to a portfolio of regional prices but can only hedge using a single liquid market instrument. An example would be ship operators exposed to fuel prices in ports around the globe and hedging this with, say, Singapore fuel oil futures or Brent crude oil forward contracts. Knowledge of regional price dynamics also allows the development of spatial optimization models for the regional chartering of a fleet of vessels which, in the case of tramp shipping, is an emerging research area.

\section{ApPEndix A. PRoOfS}

In this Appendix we present proofs of some of the results in the paper.

Proof of Proposition 1. Let $\widetilde{\epsilon}_{i}:=\sigma \delta^{-1}\left(B\left(t_{i+1}\right)-B\left(t_{i}\right)\right)$ for $i=0,1, \ldots$ From the independent increment property of Brownian motion, $\left\{\widetilde{\epsilon}_{i}\right\}_{i=0}^{\infty}$ are independent random variables. Moreover, since $B\left(t_{i+1}\right)-$ $B\left(t_{i}\right) \stackrel{d}{=} B(\delta)$, where $\stackrel{d}{=}$ mean equality in distribution, we find that $\left\{\widetilde{\epsilon}_{i}\right\}_{i=0}^{\infty}$ are IID mean-zero normally distributed random variables, with variance given by $\sigma^{2} / \delta$.

From (9) and the defintion of the polynomials $P$ and $Q$ in (6) and (7), resp., we find

$$
\delta^{-p} \Delta_{\delta}^{p} y\left(t_{i}\right) \sum_{j=1}^{p} \alpha_{j} \delta^{p-j} \Delta_{\delta}^{p-j} y\left(t_{i}\right)+\sum_{j=0}^{q} b_{j} \delta^{-j} \Delta_{\delta}^{j} \widetilde{\epsilon}_{i}
$$

Multplying with $\delta^{p}$ and using the definition of the forward differencing operator together with the fact that $t_{i}+r \delta=t_{i+r}$ for any $r \in \mathbb{N}$, yield,

$$
\begin{aligned}
y_{i+p} & +\sum_{k=1}^{p}\left(\begin{array}{l}
p \\
k
\end{array}\right)(-1)^{k} y_{i+p-k}+\sum_{j=1}^{p} \alpha_{j} \delta^{j} \sum_{k=0}^{p-j}\left(\begin{array}{c}
p-j \\
k
\end{array}\right)(-1)^{k} y_{i+p-j-k} \\
& =\sum_{j=0}^{q} b_{j} \delta^{p-j} \sum_{k=0}^{j}\left(\begin{array}{l}
j \\
k
\end{array}\right)(-1)^{k} \widetilde{\epsilon}_{i+j-k},
\end{aligned}
$$

After some algebraic (and tedious) manipulations of the terms in this expression, the result follows.

Proof of Proposition 2. From the definition of a CAR-process, $Y_{0}$ is a Gaussian process. Hence, $X$ becomes Gaussian as well since it is the integral of a Gaussian process, e.g., a limit of a sum of normally distributed random variables. The mean is trivial to compute. Concerning the variance, we find

$$
\operatorname{Var}[X(t)]=2 \int_{0}^{t} \int_{0}^{s} \operatorname{Cov}\left(Y_{0}(u), Y_{0}(s)\right) d u d s .
$$

But, when $u \leq s$,

$$
\operatorname{Cov}\left(Y_{0}(u), Y_{0}(s)\right)=\mathbb{E}\left[\int_{0}^{s} \mathbf{e}_{1}^{\top} e^{A_{0}(s-v)} \mathbf{e}_{p_{0}} d B_{0}(v) \int_{0}^{u} \mathbf{e}_{1}^{\top} e^{A_{0}(u-v)} \mathbf{e}_{p_{0}} d B_{0}(v)\right]
$$




$$
=\int_{0}^{u} \mathbf{e}_{1}^{\top} e^{A_{0}(s-v)} \mathbf{e}_{p_{0}} \mathbf{e}_{1}^{\top} e^{A_{0}(u-v)} \mathbf{e}_{p_{0}} d v,
$$

where we used the independent increment property of Brownian motion along with the Itô isometry. The result follows after some straightforward manipulations.

Proof of Proposition 3. Consider the vector-valued process $\overline{\mathbf{Z}}_{0}:=\left(X, \mathbf{Z}_{0}\right) \in \mathbb{R}^{p_{0}+1}$. By definition of the matrix $\bar{A}_{0}$, the fact that $d X(t)=Y_{0}(t) d t=\mathbf{e}_{1}^{\top} \mathbf{Z}_{0}(t) d t$, and

$$
d \mathbf{Z}_{0}(t)=A_{0} \mathbf{Z}_{0}(t) d t+\mathbf{e}_{p_{0}} \sigma_{0} d B_{0}(t)
$$

it follows

$$
d \overline{\mathbf{Z}}_{0}(t)=\bar{A}_{0} \overline{\mathbf{Z}}_{0}(t) d t+\mathbf{e}_{p_{0}+1} \sigma_{0} d B_{0}(t) .
$$

Hence, since $X(t)=\mathbf{e}_{1}^{\top}\left(X(t), \mathbf{Z}_{0}(t)\right)$, the first claim follows.

For the second, the characteristic polynomial of the matrix $\bar{A}_{0}$ is easily seen to be

$$
\bar{P}_{0}(\lambda)=\operatorname{det}\left(\bar{A}_{0}-\lambda I_{p+1}\right)=(-\lambda) P_{0}(\lambda),
$$

where $P_{0}$ is the characteristic polynomial of $A_{0}$. Hence, $\lambda=0$ is an eigenvalue of $\bar{A}_{0}$.

Proof of Proposition 4. From Proposition 1 with $q=0, p=p_{0}+1$ and CAR-matrix $\bar{A}_{0}$ defined in Proposition 3, it holds

$$
x_{i+p_{0}+1}=\sum_{k=1}^{p_{0}+1} \beta_{k} x_{i+p_{0}+1-k}+\eta_{0} \tilde{\epsilon}_{i}
$$

where $\eta_{0}=b_{0} \delta^{p_{0}+1}(-1)^{0}\left(\begin{array}{l}0 \\ 0\end{array}\right)=\delta_{p_{0}+1}$. Since $\widetilde{\epsilon}_{i}$ is Gaussian with mean zero and variance $\sigma^{2} / \delta$, we define $\epsilon_{i}:=\delta^{1 / 2} \sigma^{-1} \widetilde{\epsilon}_{i}$, which then defines a sequence of IID standard normally distributed random variables. Hence, $\eta_{0} \widetilde{\epsilon}_{i}=\sigma \delta^{p_{0}+1 / 2} \epsilon_{i}$. After some algebraic considerations, it follows that

$$
\begin{aligned}
\Delta x_{i+p_{0}}= & x_{i+p_{0}+1}-x_{i+p_{0}} \\
= & \left(\beta_{1}-1\right) \Delta x_{i+p_{0}-1}+\left(\beta_{1}-1+\beta_{2}\right) \Delta x_{i+p_{0}-2}+\cdots+\left(\beta_{1}-1+\beta_{2}+\cdots+\beta_{p_{0}}\right) \Delta x_{i} \\
& \quad+\left(\beta_{1}-1+\beta_{2}+\cdots+\beta_{p+1}\right) x_{i}+\sigma \delta^{p_{0}+1 / 2} \epsilon_{i} .
\end{aligned}
$$

Consider the term in front of $x_{i}$ above, that is, $\sum_{k=1}^{p_{0}+1} \beta_{k}-1$. From the definition of $\beta_{k}$, we find

$$
\sum_{k=1}^{p_{0}+1} \beta_{k}=\sum_{k=1}^{p_{0}+1}(-1)^{k+1}\left(\begin{array}{c}
p_{0}+1 \\
k
\end{array}\right)-\sum_{k=1}^{p_{0}+1} \sum_{j=1}^{k} \alpha_{j} \delta^{j}(-1)^{k-j}\left(\begin{array}{c}
p_{0}+1-j \\
k-j
\end{array}\right) .
$$

But we know that $(x+y)^{n}=\sum_{k=0}^{n}\left(\begin{array}{l}n \\ k\end{array}\right) x^{k} y^{n-k}$, so with $x=-1, y=1$

$$
0=\sum_{k=0}^{p_{0}+1}(-1)^{k}\left(\begin{array}{c}
p_{0}+1 \\
k
\end{array}\right)=\left(\begin{array}{c}
p_{0}+1 \\
0
\end{array}\right)+\sum_{k=1}^{p_{0}+1}\left(\begin{array}{c}
p_{0}+1 \\
k
\end{array}\right)(-1)^{k}
$$

and we conclude $\sum_{k=1}^{p_{0}+1}(-1)^{k+1}\left(\begin{array}{c}p_{0}+1 \\ k\end{array}\right)=1$. Therefore,

$$
\begin{aligned}
1-\sum_{k=1}^{p_{0}+1} \beta_{k} & =\sum_{k=1}^{p_{0}+1} \sum_{j=1}^{k} \alpha_{j} \delta^{j}(-1)^{k-j}\left(\begin{array}{c}
p_{0}+1-j \\
k-j
\end{array}\right) \\
& =\sum_{k=1}^{p_{0}} \sum_{j=1}^{k} \alpha_{j} \delta^{j}(-1)^{k-j}\left(\begin{array}{c}
p_{0}+1-j \\
k-j
\end{array}\right)+\sum_{j=1}^{p_{0}} \alpha_{j} \delta^{j}(-1)^{p_{0}+1-j}\left(\begin{array}{c}
p_{0}+1-j \\
p_{0}+1-j
\end{array}\right)
\end{aligned}
$$

where we used that $\alpha_{p_{0}+1}=0$ in the last equality. But we see that

$$
\begin{aligned}
1-\sum_{k=1}^{p_{0}+1} \beta_{k}= & \alpha_{1} \delta\left(\sum_{k=0}^{p_{0}}(-1)^{k}\left(\begin{array}{c}
p_{0} \\
k
\end{array}\right)\right)+\alpha_{2} \delta^{2}\left(\sum_{k=0}^{p_{0}-1}(-1)^{k}\left(\begin{array}{c}
p_{0}-1 \\
k
\end{array}\right)\right)+ \\
& \cdots+\alpha_{p_{0}} \delta^{p_{0}}\left(\sum_{k=0}^{1}(-1)^{k}\left(\begin{array}{l}
1 \\
k
\end{array}\right)\right) \\
=0 &
\end{aligned}
$$


The claimed integrated $\operatorname{AR}\left(p_{0}\right)$ dynamics follows.

Proof of Proposition 5: First, by $\mathcal{F}_{t}$-measurability, it follows

$$
F_{X}(t, T)=\mathbb{E}_{Q}\left[\exp \left(\int_{0}^{T} Y_{0}(s) d s\right) \mid \mathcal{F}_{t}\right]=S(t) \mathbb{E}_{Q}\left[\exp \left(\int_{t}^{T} Y_{0}(s) d s\right) \mid \mathcal{F}_{t}\right]
$$

From the $Q$-dynamics of $\mathbf{Z}_{0}$ in (23) we find for $t \leq s$,

$$
\mathbf{Z}_{0}(s)=e^{A_{0}(s-t)} \mathbf{Z}_{0}(t)-\sigma_{0} \gamma_{0} A_{0}^{-1}\left(e^{A_{0}(s-t)}-I_{p_{0}}\right) \mathbf{e}_{p_{0}}+\int_{t}^{s} e^{A_{0}(s-u)} \mathbf{e}_{p_{0}} d W_{0}(u) .
$$

Hence, applying the stochastic Fubini theorem,

$$
\begin{aligned}
\int_{t}^{T} Y_{0}(s) d s=\mathbf{e}_{1}^{\top} A_{0}^{-1}\left(e^{A_{0}(T-t)}-I_{p_{0}}\right) \mathbf{Z}_{0}(t)-\sigma_{0} \gamma_{0} \mathbf{e}_{1}^{\top} A_{0}^{-2}\left(e^{A_{0}(T-t)}-I_{p_{0}}\right) \mathbf{e}_{p_{0}} \\
+\sigma_{0} \gamma_{0} \mathbf{e}_{1}^{\top} A_{0}^{-1} \mathbf{e}_{p_{0}}(T-t)+\sigma_{0} \int_{t}^{T} \mathbf{e}_{1}^{\top} A_{0}^{-1}\left(e^{A_{0}(T-u)}-I_{p_{0}}\right) \mathbf{e}_{p_{0}} d W_{0}(u) .
\end{aligned}
$$

The result follows after appealing to the $\mathcal{F}_{t}$-measurability of $\mathbf{Z}_{0}(t)$ and the fact that the stochastic integral above is independent of $\mathcal{F}_{t}$ due to the independent increment property of Brownian motion. Furthermore, the stochastic integral is Gaussian distributed with mean zero and variance given by the Itô isometry.

Proof of Proposition 6: We observe by definition of $F_{X}(t, T)$ that $t \mapsto F_{X}(t, T)$ is a $Q$-martingale for $t \leq T$. Using Itô's Formula, it is thus only the term giving a $d W_{0}$-contribution that matters, which is the $p_{0}$-coordinate of $\mathbf{Z}_{0}$. Hence, differentiating the function $h(\mathbf{z}, s)$ in Proposition 5 with respect to the $p_{0}$ coordinate we find

$$
d F_{X}(t . T)=F_{X}(t, T)\left(\mathbf{e}_{1}^{\top} A_{0}^{-1}\left(e^{A_{0}(T-t)}-I_{p_{0}}\right) \mathbf{e}_{p_{0}}\right) \sigma_{0} d W_{0}(t)
$$

and the Proposition follows.

Proof of Corollary 7: Obviously, $\Sigma_{X}(0)=0$ since $\exp \left(A_{0} 0\right)=I_{p_{0}}$. Moreover, since $A_{0}$ is assumed to have eigenvalues with negative real part, it follows that $\lim _{s \rightarrow \infty} e^{A_{0} s} \mathbf{z}=0$ for all $\mathbf{z} \in \mathbb{R}^{p_{0}}$. Therefore, $\lim _{s \rightarrow \infty} \Sigma_{X}(s)=-\sigma_{0} \mathbf{e}_{1}^{\top} A_{0}^{-1} \mathbf{e}_{p_{0}}$. From Benth and Šaltyte Benth [12, Lemma 4.2], we have $A_{0}^{-1}$ explicitly available and it follows

$$
\mathbf{e}_{1}^{\top} A_{0}^{-1}=\left(-\alpha_{p_{0}-1} / \alpha_{p_{0}},-\alpha_{p_{0}-2} / \alpha_{p_{0}}, \ldots,-\alpha_{1} / \alpha_{p_{0}},-1 / \alpha_{p_{0}}\right) .
$$

Hence, $\mathbf{e}_{1}^{\top} A_{0}^{-1} \mathbf{e}_{p_{0}}=-1 / \alpha_{p_{0}}$ and the proof is complete.

Proof of Proposition 8. Fix $i=1, \ldots, n$, and recall that $S_{i}(t)=\exp \left(X(t)+\theta_{i}+Y_{i}(t)\right)$. From (26) we have for $T \geq t$ that

$$
Y_{i}(T)=\mathbf{e}_{1}^{\top} e^{A_{i}(T-t)} \mathbf{Z}_{i}(t)-\sigma_{i} \gamma_{i} \mathbf{e}_{1}^{\top} A_{i}^{-1}\left(e^{A_{i}(T-t)}-I_{p_{i}}\right) \mathbf{e}_{p_{i}}+\sigma_{i} \int_{t}^{T} \mathbf{e}_{1}^{\top} e^{A_{i}(T-s)} \mathbf{e}_{p_{i}} d W_{i}(s) .
$$

We note that $\mathbf{Z}_{i}(t)$ is $\mathcal{F}_{t}$-measurable and the stochastic integral with respect to $W_{i}$ is $\mathcal{F}_{t}$-independent since Brownian motion has independent increments. Using the same line of arguments as in the proof of Proposition 5 yields,

$$
\begin{aligned}
& F_{i}(t, T) \\
&= S(t) \exp \left(\mathbf{e}_{1}^{\top} A_{0}^{-1}\left(e^{A_{0}(T-t)}-I_{p_{0}}\right) \mathbf{Z}_{0}(t)+\mathbf{e}_{1}^{\top} e^{A_{i}(T-t)} \mathbf{Z}_{i}(t)\right) \\
& \times \exp \left(-\sigma_{0} \gamma_{0} \mathbf{e}_{1}^{\top} A_{0}^{-2}\left(e^{A_{0}(T-t)}-I_{p_{0}}\right) \mathbf{e}_{p_{0}}+\sigma_{0} \gamma_{0} \mathbf{e}_{1}^{\top} A_{0}^{-1} \mathbf{e}_{p_{0}}(T-t)\right) \\
& \times \exp \left(-\sigma_{i} \gamma_{i} \mathbf{e}_{1}^{\top} A_{i}^{-1}\left(e^{A_{i}(T-t)}-I_{p_{i}}\right) \mathbf{e}_{p_{i}}\right) \\
& \times \mathbb{E}_{Q}\left[\exp \left(\sigma_{0} \int_{t}^{T} \mathbf{e}_{1}^{\top} A_{0}^{-1}\left(e^{A_{0}(T-s)}-I_{p_{0}}\right) \mathbf{e}_{p_{0}} d W_{0}(s)+\sigma_{i} \int_{t}^{T} \mathbf{e}_{1}^{\top} e^{A_{i}(T-s)} \mathbf{e}_{p_{i}} d W_{i}(s)\right) \mid \mathcal{F}_{t}\right] .
\end{aligned}
$$


MULTIVARIATE MODELING AND ANALYSIS OF REGIONAL OCEAN FREIGHT RATES

Let $\rho_{0, i}$ be the correlation coefficient between the two Brownian motions $W_{0}$ and $W_{i}$. Then, since the sum of the two stochastic integrals is mean zero Gaussian distributed, we find

$$
\begin{aligned}
\mathbb{E}_{Q}\left[\exp \left(\sigma_{0} \int_{t}^{T} \mathbf{e}_{1}^{\top} A_{0}^{-1}\left(e^{A_{0}(T-s)}-I_{p_{0}}\right) \mathbf{e}_{p_{0}} d W_{0}(s)+\sigma_{i} \int_{t}^{T} \mathbf{e}_{1}^{\top} e^{A_{i}(T-s)} \mathbf{e}_{p_{i}} d W_{i}(s)\right) \mid \mathcal{F}_{t}\right] \\
=\exp \left(\frac{1}{2} \int_{0}^{T-t} \sigma_{0}^{2}\left(\mathbf{e}_{1}^{\top} A_{0}^{-1}\left(e^{A_{0} s}-I_{p_{0}}\right) \mathbf{e}_{p_{0}}\right)^{2}+2 \rho_{0, i} \sigma_{0} \sigma_{i}\left(\mathbf{e}_{1}^{\top} A_{0}^{-1}\left(e^{A_{0} s}-I_{p_{0}}\right) \mathbf{e}_{p_{0}}\right)\left(\mathbf{e}_{1}^{\top} e^{A_{i} s} \mathbf{e}_{p_{i}}\right)\right. \\
\left.\quad+\sigma_{i}^{2}\left(\mathbf{e}_{1}^{\top} e^{A_{i} s} \mathbf{e}_{p_{i}}\right)^{2} d s\right) .
\end{aligned}
$$

Collecting terms yields the result.

\section{REFERENCES}

[1] Adland, R., Bjerknes, F. and Herje, C. (2017). Spatial efficiency in the bulk freight market, Maritime Policy and Management, 44(4), pp. 413-425. doi: 10.1080/03088839.2017.1298864

[2] Alizadeh, A. and Nomikos, N. (2009). Shipping Derivatives and Risk Management, Palgrave: London

[3] Applebaum, D. (2009). Lévy Processes and Stochastic Calculus, Cambridge University Press: Cambridge.

[4] Bachmeier, L.J. and Griffin, J.M. (2006). Testing for market integration: Crude oil, coal and natural gas, The Energy Journal, 27(2), pp. 55-71.

[5] Ballotta, L., and Bonfiglioli, E. (2016). Multivariate asset models using Levy processes and applications. European Journal of Finance, 22, pp. 1320-1350.

[6] Baltic Exchange (2016). Guide to market benchmarks, Version 2.1, January, Baltic Exchange Information Services Ltd: London, UK.

[7] Barndorff-Nielsen, O. E. (1977). Exponentially decreasing distributions for the logarithm of particle size. Proceedings of the Royal Society of London A, 353, pp. 401-419.

[8] Barndorff-Nielsen, O. E. (1998). Processes of normal inverse Gaussian type. Finance and Stochastics, 2, pp. 41-68.

[9] Barndorff-Nielsen, O. E., and Shephard, N. (2001). Non-Gaussian Ornstein-Uhlenbeck-based models and some of their uses in economics. Journal of the Royal Statistical Society B, 63(2), pp. 167-241 (with discussion).

[10] Benth, F. E. (2011). The stochastic volatility model of Barndorff-Nielsen and Shephard in commodity markets. Mathematical Finance, 21(4), pp. 595-625.

[11] Benth, F. E. (2010). On forward price modelling in power markets. In Alternative Investments and Strategies, R. Kiesel, M. Scherer and R. Zagst (eds.), World Scientific, Ch.5, pp. 93122.

[12] Benth, F. E., and Šaltytė Benth, J. (2013). Modeling and Pricing in Financial Markets for Weather Derivatives, World Scientific.

[13] Benth, F. E., Šaltytė Benth, J., and Koekebakker, S. (2008). Stochastic Modelling of Electricity and Related Markets, World Scientific.

[14] Benth, F.E., and Dethering, N. (2015). Pricing and hedging Asian-style options in energy. Finance \& Stochastics, 19(4), pp. 849889.

[15] Benth, F. E. and Koekebakker, S. (2015). Pricing of forwards and other derivatives in cointegrated commodity markets, Energy Economics, 52, pp. 104-117.

[16] Berg-Andreassen, J.A. (1996), Some properties of international maritime statistics. Maritime Policy and Management, 23(4), pp. 381-395.

[17] Berg-Andreassen, J.A. (1997), Efficiency and interconnectivity in international shipping markets. International Journal of Transport Economics, XXIV(2), pp. 241-257.

[18] Brockwell, P.J. (2001). Lévy-driven CARMA processes. Ann. Inst. Statist. Math., 53, pp. 113-124.

[19] Chang, Y., Miller, J.I., and Park, J.Y. (2009), Extracting a Common Stochastic Trend: Theory and Some Applications, Journal of Econometrics, 150, pp. 231-247.

[20] Clarkson Research (2016), Shipping Intelligence Network, www.clarksons.net

[21] Dickey, D. A. and Fuller, W. A. (1979). Distribution of the estimators for autoregressive time series with a unit root. Journal of the American Statistical Association, 74(366), pp. 427-431.

[22] Duffie, D., and Richardson, H. R. (1991). Mean-variance hedging in continuous time. Annals Appl. Prob., 1(1), pp. 1-15.

[23] Eberlein, E., and Keller, U. (1995). Hyperbolic distributions in finance. Bernoulli, 1, pp. 281-299.

[24] Elliott, G., Rothenberg, T. J. and Stock, J. H. (1996). Efficient tests for an autoregressive unit root, Econometrica, 64, pp. 813836.

[25] Engle, R. F. and Granger, C. W. J. (1987). Co-integration and error correction: Representation, estimation and testing. Econometrica, 55, pp. 251-276.

[26] Glen, D, and Rogers, P. (1997), Does weight matter? A statistical analysis of the SSY Capesize index. Maritime Policy and Management, 24(4), pp. 351-364.

[27] Hummels, D.L. and Schaur, G. (2013). Time as a Trade Barrier, American Economic Review, 103(7), pp. 2935-2959.

[28] Johansen, S. (1991). Estimating and testing cointegration vectors in Gaussian vector autoregressive models. Econometrica, 59(6), pp. 1551-1580.

[29] Karatzas, I., and Shreve, S. E. (1994). Brownian Motion and Stochadstic Calculus, Springer Verlag. 
[30] Kavussanos, M. G., and Alizadeh, A. (2001). Seasonality patterns in dry bulk shipping spot and time charter freight rates. Transportation Research Part E, 37, 443-467.

[31] Ko, B.-W. (2011a), An Application of Dynamic Factor Model to Dry Bulk Market - Focusing on the Analysis of Synchronicity and Idiosyncrasy in the Sub-Markets with Different Ship Size, KMI International Journal of Maritime Affairs and Fisheries, 3(1), pp. 69-82.

[32] Ko, B.-W. (2011b), Dynamics of Dry Bulk Freight Market: Through the Lens of a Common Stochastic Trend Model, The Asian Journal of Shipping and Logistics, 27(3), pp. 387-404.

[33] Kyriakou, I., Pouliasis, P. K., Papapostolou, N. C. and Andriosopoulos, K. D. (2017). Freight derivatives pricing for decoupled mean-reverting diffusion and jumps. Available at SSRN: https://ssrn.com/abstract=2970991

[34] Lanza, A., Manera, M. and Giovannini, M. (2005), Modeling and forecasting cointegrated relationships among heavy oil and products prices, Energy Economics, 27, pp. 831-848.

[35] Ng, S. and Perron P. (2001). Lag length selection and the construction of unit root tests with good size and power, Econometrica, 69, pp. 1519-1554.

[36] Nomikos, N. K., Kyriakou, I., Papapostolou, N. C., Pouliasis, P. K. (2013). Freight options: Price modelling and empirical analysis. Transportation Research Part E: Logistics and Transportation Review, 51, pp. 82-94.

[37] Øigård, T. A., and Hanssen, A. (2002).The multivariate normal inverse Gaussian heavy-tailed distribution: Simulation and estimation. In IEEE International Conference on Acoustics, Speech, and Signal Processing, Orlando, FL, USA, Vol. 2, pp. 14891492.

[38] Papiez, M. and Smiech, S. (2015), Dynamic steam coal market integration: Evidence from rolling cointegration analysis, Energy Economics, 51, pp. 510-520.

[39] Pirrong, C. (2014).The Economics of Commodity Trading Firms. Trafigura.

[40] Prokopczuk, M. (2011). Pricing and hedging in the freight futures market. Journal of Futures Markets, 31(5), pp. 440-464.

[41] Rydberg, T. H. (1997). The normal inverse Gaussian Lévy process: Simulation and approximation. Communications in Statistics: Stochastic Models, 34, pp. 887-910.

[42] Said, S.E. and Dickey D.A. (1984). Testing for unit roots in autoregressive-moving average models of unknown order, Biometrika, 71, pp. 599-608.

[43] Schwartz, E. and Smith, J. (2000). Short-term variations and long-term dynamics in commodity prices. Management Science, 46, pp. :893-911.

[44] Siliverstovs, B., LHegaret, G., Neumann, A. and von Hirschhausen, C. (2005), International market integration for natural gas? A cointegration analysis of prices in Europe, North America and Japan, Energy Economics, 27(4), pp. 603-615.

[45] Stock, J. H., and Watson, M. W. (1988). Testing for common trends. Journal of the American Statistical Association, 83, pp. 1097-1107.

[46] Stopford, M. (2009). Maritime Economics. 3rd ed. London: Routledge.

[47] Veenstra, A. and Franses, P.H. (1997), A cointegration approach to forecasting freight rates in the dry bulk shipping sector, Transportation Research Part A, 31(6), pp. 447-458.

[48] Wårell, L. (2006), Market integration in the international coal industry: A cointegration approach, The Energy Journal, 27(1), pp. $99-118$. 